Groupe d'Annecy

Groupe de Lyon

Laboratoire

d'Annecy-le-Vieux de

Physique des Particules

Ecole Normale

Supérieure de Lyon

\title{
CASCADE PARTICLES, NUCLEAR EVAPORATION, AND RESIDUAL NUCLEI IN HIGH ENERGY HADRON-NUCLEUS INTERACTIONS
}

\author{
A. Ferrari, P.R. Sala \\ INFN, Sezione di Milano, Via Celoria 16, I-20133 Milano, Italy \\ J. Ranft \\ Laboratoire de Physique Théorique ENSLAPP用 \\ Groupe d'Annecy: LAPP, Chemin de Bellevue, BP 110, \\ F-74941 Annecy-le-Vieux Cedex, France. \\ and \\ S. Roesler \\ Universität Siegen, Fachbereich Physik, D-57068 Siegen, Germany.
}

\begin{abstract}
Based on a Monte Carlo realization of the Dual Parton Model we study the production of target associated particles and of nuclear fragments in high energy hadron-nucleus interactions. A formation zone intranuclear cascade of low energy secondaries inside the target nucleus is discussed. We calculate excitation energies of residual nuclei left after the intranuclear cascade process and treat their further disintegration by introducing models for the evaporation of protons, neutrons, and light fragments, high energy fission, and by applying a Fermi Break-up model to light nuclear fragments. The results are compared to data on target associated particle production. We furthermore calculate cross sections for the production of nuclear fragments.

ENSLAPP-A-551/95

Siegen SI 95-09

September 1995
\end{abstract}

${ }^{*}$ URA 14-36 du CNRS, associée à l'Ecole Normale Supérieure de Lyon et à l'Université de Savoie. 


\section{Introduction}

The Dual Parton Model (DPM) [1] and Monte Carlo (MC) implementations of this model for hadron-hadron [2, 3], hadron-nucleus, and nucleus-nucleus collisions [4, 5] have been quite successful in describing many aspects of hadron production in high energy collisions. So far however, MC models for hadron-nucleus and nucleus-nucleus collisions based on the DPM did mainly describe the high energy component of newly created hadrons, not the many particles resulting from the nuclear disintegration following the high energy collisions.

Models for nuclear evaporation and fragmentation and for high energy fission are however usually included in hadron cascade models such as FLUKA [6, 7, 8] used for detector simulation and for the evaluation of radiation damage to high energy accelerator and detector components. In the older models, the nuclear excitation energy, which is the starting point for the calculation of the nuclear disintegration, was often introduced only in a phenomenological way [9] or it was calculated on the basis of valid but simple intranuclear cascade models [10, 11] which are not applicable in the multi-GeV energy range of present experiments. The intranuclear cascade models have been greatly improved since then [6, 7, 8, 12] and their range of validity can be extended to higher energies due to the introduction of the formation zone concept [13, 14].

Here, we use the DTUNUC [4] and DPMJET-II [5] MC implementations of the DPM for high energy hadron-nucleus and nucleus-nucleus collisions. These MC models contain a formation zone intranuclear cascade which is responsible for knocking out cascade protons and neutrons of the residual nucleus. The cascade protons have energies which are typical for the so called grey prongs observed in emulsion experiments. Therefore, we are able to calculate the nuclear excitation energy of the residual nucleus. In a second step, this excitation energy is the basis for nuclear evaporation, and high energy fission reactions. In the present paper these mechanisms are investigated in hadron-nucleus collisions. In a forthcoming publication we intend to extend these studies to peripheral high energy nucleus-nucleus collisions.

In Sec.2 we describe the formation zone intranuclear cascade model 15 and focus on the calculation of excitation energies. In Sec.3 models for evaporation and fragmentation are presented. In Sec.4 we compare computed cross sections and multiplicities of grey and black prong production to experimental data. Furthermore, cross sections for the production of residual nuclei are discussed. In Sec.5 we summarize our results.

\section{The calculation of excitation energies in the forma- tion zone intranuclear cascade model}

\subsection{The two-component Dual Parton Model for hadron-nucleus and nucleus-nucleus collisions}

The two-component DPM and its MC realizations have been discussed in detail in [1, 2, 3, 4, 5, 16]. Therefore, we summarize only briefly the main steps leading to the multiparticle state, which is the starting point for the intranuclear cascade and evaporation models being described in this paper. 
The MC model for hadron-nucleus and nucleus-nucleus interactions starts from an impulse approximation for the nucleons of the interacting nuclei. The spatial initial configuration, i.e. the positions of the nucleons in space-time in the rest system of the corresponding nucleus, is sampled from standard density distributions. For energies above $3-5 \mathrm{GeV} /$ nucleon the collision proceeds via $\nu$ elementary interactions between $\nu_{p}$ and $\nu_{t}$ nucleons from the projectile and target, resp. The values $\nu, \nu_{p}$, and $\nu_{t}$ are sampled according to Glauber's multiple scattering formalism using the $\mathrm{MC}$ algorithm of [17]. The particle production is well described by the two-component DPM which is applied as in hadron-hadron interactions [2, 16, 18]. As a result a system of chains connecting partons of the nucleons involved in the scattering process is formed. The chains are hadronized applying the model JETSET [19, 20]. The hadrons may than cause intranuclear cascade processes, which are treated by the formation zone intranuclear cascade model [15], an extension of the intranuclear cascade model [10, 11]. At energies below 3-5 GeV/nucleon the formation zone intranuclear cascade model itself provides a reasonable description of inelastic nuclear collisions.

In the following we summarize the main ideas of the formation zone intranuclear cascade model for hadron-nucleus interactions. Modifications which have to be introduced to describe nucleus-nucleus collisions will be discussed in a forthcoming paper. The physical picture explaining the absence of the intranuclear cascade at high energies is the concept of the formation zone [13]. It has been introduced in analogy to the Landau-Pomeranchuk [21] effect, which explains the observation that electrons passing through material become more penetrating at high energies. For the formation zone of an electron with 4-momentum $p$ and energy $E$ upon radiation of a photon with 4-momentum $k$ one obtains

$$
\tau=\frac{E}{k \cdot p}=\frac{E}{m} \frac{1}{\omega_{e}}
$$

where $\omega_{e}$ is the frequency of the photon in the rest frame of the electron and $E / m$ is the time dilatation factor from the electron rest frame to the laboratory. Within the quark model, the states being formed in the primary nucleon-nucleon interaction can be understood as consisting of valence quarks only, i.e without the full system of sea quarks, antiquarks, and gluons and have therefore a reduced probability for hadronic interactions inside the nucleus [15]. The formation zone concept can be translated to hadron production as follows [14. Denoting the 4-momenta of the projectile hadron $p_{p}$ and the secondary hadron $p_{s}$ in the laboratory frame with

$$
p_{p}=\left(E_{p}, 0,0, \sqrt{E_{p}^{2}-m_{p}^{2}}\right), \quad p_{s}=\left(E_{s}, \vec{p}_{s \perp}, \sqrt{E_{s}^{2}-m_{s}^{2}-\vec{p}_{s \perp}^{2}}\right)
$$

and replacing in Eq. (1) the electron momentum by $p_{p}$ and the photon momentum by $p_{s}$, the hadron formation zone reads for $E_{p} \gg m_{p}$

$$
\tau_{\text {Lab }}=\frac{2 E_{s}}{\left(m_{p} x\right)^{2}+m_{s}^{2}+p_{s \perp}^{2}}, \quad x=\frac{E_{s}}{E_{p}} .
$$

Since for most of the produced secondaries the term $\left(m_{p} x\right)^{2}$ can be neglected one can approximate

$$
\tau_{\mathrm{Lab}} \approx \gamma_{s} \tau_{s}, \quad \gamma_{s}=\frac{E_{s}}{m_{s}}
$$


In the rest system of the secondary hadron $s$, we define an average formation time $\tau_{s}$ needed to create a complete hadronic state [15, 14

$$
\tau_{s}=\tau_{0} \frac{m_{s}^{2}}{m_{s}^{2}+p_{s \perp}^{2}} .
$$

$\tau_{0}$ is a free parameter, which has to be determined by comparing particle production within the model to experimental data. Typical values are in the range from $1 \mathrm{fm} / c$ to $10 \mathrm{fm} / \mathrm{c}^{\mathbb{f}}$. From the comparisons discussed further below we find $\tau_{0}=2 \mathrm{fm} / c$. For each secondary we sample a formation time $\tau$ from an exponential distribution [22] with an average value as given in Eq. (5). As it was described in [四, in our MC model we know the full space-time history of the collision. In any particular Lorentz frame we can follow the trajectories of the secondaries created in the hadronization of the chains in space and time. Due to relativistic time dilatation secondaries with high energies in the nucleus rest system are mostly formed outside the nucleus and are therefore not able to initiate intranuclear cascade processes. On the other hand, the lower the energy of the secondary hadronic system the higher is the probability to form a hadron inside the nucleus. These hadrons may therefore reinteract with spectator nucleons taking into account the nuclear geometry. In the model, inelastic secondary interactions of hadrons with energies below $9 \mathrm{GeV}$ are described with the code HADRIN [23]. In general, the intranuclear cascade would start with resonances resulting from the chain hadronization procedure, but we apply the following way. Since the interaction cross sections of resonances needed within HADRIN are less well known, we firstly treat their decay and sample the intranuclear cascade starting from the stable particles. We assume that the effect of this approximation can mostly be compensated by using an effective $\tau_{0}$ value. Reinteractions within the colliding nucleus beyond $9 \mathrm{GeV}$ are very rare and therefore neglected in the present approach. Pauli's principle is taken into account as described in [4]. For the secondaries produced in intranuclear cascade processes we apply the same formalism as described above and therefore obtain a formation zone intranuclear cascade in all generations of secondaries.

\subsection{The calculation of nuclear excitation energies}

The treatment of nuclear effects within the MC model has already been discussed in [⿴囗. . Since they are essential in calculating excitation energies of nuclei left after primary interactions and intranuclear cascade processes we summarize the basic ideas. Fermi momenta for nucleons as well as a simplified treatment of the nuclear potential are applied to control the generation of low-energy particles. Nucleon momenta are sampled from zero-temperature Fermi distributions

$$
\frac{d N^{\mathrm{n}, \mathrm{p}}}{d p}=N^{\mathrm{n}, \mathrm{p}} \frac{3 p^{2}}{\left(p_{\mathrm{F}}^{\mathrm{n}, \mathrm{p}}\right)^{3}}
$$

\footnotetext{
${ }^{2}$ In Refs. [4, 5] $\tau_{0}$ was fixed to $\tau_{0}=5 \mathrm{fm} / c$ whereas in Ref. [15 $\tau_{0}=1-2 \mathrm{fm} / c$ was used.
} 
Here and in the following the indices "n" and "p" denote neutrons and protons, resp. The maximum allowed Fermi momenta of neutrons and protons are

$$
p_{\mathrm{F}}^{\mathrm{n}, \mathrm{p}}=\left[\left(\frac{N^{\mathrm{n}, \mathrm{p}}}{V_{A}}\right) \frac{3 h^{3}}{8 \pi}\right]^{\frac{1}{3}}
$$

with $V_{A}$ being the volume of the corresponding nucleus with an approximate nuclear radius $R_{A}=r_{0} A^{1 / 3}, r_{0}=1.29 \mathrm{fm}$. Modifications of the actual nucleon momentum distribution, as they would arise, for instance, taking the reduced density and momenta in the nuclear skin into consideration, effectively result in a reduction of the Fermi momenta as compared to those sampled from Eq. (7). This effect can be estimated by a correction factor $\alpha_{\text {mod }}^{\mathrm{F}}$ which modifies the Fermi-momenta. Results presented in this paper have been obtained with $\alpha_{\text {mod }}^{\mathrm{F}}=0.75$. The depth of the nuclear potential is assumed to be the Fermi energy and the binding energy for outer shell nucleons

$$
V^{\mathrm{n}, \mathrm{p}}=\frac{\left(p_{\mathrm{F}}^{\mathrm{n}, \mathrm{p}}\right)^{2}}{2 m_{\mathrm{n}, \mathrm{p}}}+E_{\text {bind }}^{\mathrm{n}, \mathrm{p}} .
$$

To extend the applicability of the model to the energy region well below $1 \mathrm{GeV}$ an approximate treatment of the Coulomb-potential is provided. The Coulomb-barrier modifying the nuclear potential is calculated from

$$
V_{\mathrm{C}}=\frac{e^{2}}{4 \pi \epsilon_{0} r_{0}} \frac{Z_{1} Z_{2}}{\left(A_{1}^{1 / 3}+A_{2}^{1 / 3}\right)}
$$

with the mass numbers $A_{1}, A_{2}$ and charges $Z_{1}, Z_{2}$ of the colliding nuclei, i.e. with $A_{1}=\left|Z_{1}\right|=1$ for charged hadrons entering or leaving the target nucleus. $e$ denotes the elementary charge and $r_{0}=1.29 \mathrm{fm}$.

The excitation energy $U$ of the residual nucleus with mass number $A_{\text {res }}$ and charge $Z_{\text {res }}$, i.e. the energy above the ground state mass $E_{0, \text { res }}$, is given as

$$
\begin{aligned}
U & =E_{\text {res }}-E_{0, \text { res }}, \\
E_{0, \text { res }} & =Z_{\text {res }} m_{\mathrm{p}}+\left(A_{\text {res }}-Z_{\text {res }}\right) m_{\mathrm{n}}-E_{\text {bind }}\left(A_{\text {res }}, Z_{\text {res }}\right) .
\end{aligned}
$$

We calculate the binding energy $E_{\text {bind }}\left(A_{\text {res }}, Z_{\text {res }}\right)$ using the experimentally determined excess masses of all known (measured) nuclides and using mass formulae for nuclides far from the stable region, where no measurements are available. The excitation energy is obtained within our model from an explicit consideration of the effects of the nuclear potential (Eq. (8)) and the Coulomb energy (Eq. (9)), i.e. from corrections which are applied to the 4-momenta of the final state hadrons leaving the spectator nucleus. We modify the energies of these hadrons by the potential barrier and rescale the 3-momenta correspondingly. It is assumed that these corrections have to be applied to nucleons wounded in primary and secondary interactions and to those hadrons only, which are formed inside the spectator nucleus corresponding to the sampled formation time. Among these particles we find apart from the nucleons a small fraction of other baryons, which are assumed to move in a nucleon potential and mesons to which we apply an effective meson potential of $0.002 \mathrm{GeV}$. Due to energy-momentum conservation these corrections lead to a recoil momentum and, therefore, to an excitation of the residual nucleus. In addition, there is a further contribution to the recoil momentum of the 
residual nucleus arising from potential corrections applied to the momentum of the projectile hadron entering the nuclear potential and from cascade nucleons with kinetic energies below the nuclear potential which are therefore not able to escape the spectator nucleus.

In Fig. 1. 1a we show the dependence of the average excitation energies of the target residual nuclei on the momenta of the projectile in the laboratory. The decrease of the excitation energy for momenta below about $50 \mathrm{GeV} / c$ is mainly due to the breakdown of the Glauber cascade ${ }^{[}$as it can be clearly seen for $\mathrm{Au}$ and $\mathrm{Pb}$ targets. This is not the case for light nuclei where even at high energies only up to 2-3 target nucleons are involved on average in the primary interaction. This threshold behaviour will be discussed with respect to experimental information on grey and heavy particles in more detail further below. At high energies the average excitation energies are almost independent on the projectile momentum as one would expect from limiting fragmentation [24]. The average excitation energies per nucleon of the residual target nucleus are given in Fig.1 $1 \mathrm{~b}$. In difference to Fig.17a the $p_{\text {Lab-dependence is }}$ similar for all target nuclei apart from a constant shift towards higher excitation energies per nucleon for light nuclei. This is due to a smaller ratio of wounded nucleons to all target nucleons for heavy nuclei as compared to light nuclei.

In Fig.2 we show the average excitation energy of the residual target nucleus depending on the mass number of the target for proton-nucleus interactions at $300 \mathrm{GeV} / c$. The different symbols correspond to several numbers of nucleons lost by the target in primary and secondary interactions, i.e. $\Delta A$ is defined by $\Delta A=A-A_{\text {res }}$. The excitation energy is strongly correlated to the number of removed nucleons. The more nucleons one removes from the target, the more energy is deposited into the spectator nucleus. For a fixed number $\Delta A$ the excitation energy is increasing with the mass number of the target. The reason for this is, that in heavy targets we need more cascading to remove a given number of nucleons than in light targets. As an example, the distribution of excitation energies and of excitation energies per nucleon of the target prefragment are shown for proton-gold interactions at $300 \mathrm{GeV} / c$ in Fig. 3 a,b. In addition to the distributions obtained taking all prefragments into account (labelled "all $A_{\text {res }}$ ) we give distributions which correspond to several mass ranges, in particular to several lower cuts in the prefragment mass. Again, the more nucleons are involved in the primary interaction and the intranuclear cascade the higher are the mean excitation energies and, therefore, the broader are the excitation energy distributions.

\section{Evaporation/Fragmentation}

At the end of the intranuclear cascade the residual nucleus is supposed to be left in an equilibrium state, in which the excitation energy $U$ is shared by a large number of nucleons. Such an equilibrated compound nucleus is supposed to be characterized by its mass, charge, and excitation energy with no further memory of the steps which led to its formation. The excitation energy can be higher than the separation energy, thus nucleons and light fragments

\footnotetext{
${ }^{3}$ Note, that the Glauber cascade as obtained with Glauber's formalism is biased by sampling the actual chain systems [4. In order to ensure that the chain masses $M_{\text {chain }}^{2}=s x_{p} x_{t}$ exceed the masses of the lowestmass hadronic states with the corresponding quantum numbers lower $x$-cuts are imposed for all parton systems. Therefore, at low energies these $x$-cuts may reduce the number of sea quark containing chains.
} 
$\left(\alpha, \mathrm{d},{ }^{3} \mathrm{H},{ }^{3} \mathrm{He}\right)$ can still be emitted: they constitute the low-energy (and most abundant) part of the emitted particles in the rest system of the residual nucleus, having an average energy of few $\mathrm{MeV}$. The emission process can be well described as an evaporation from a hot system. The treatment starts from the formula of Weisskopf [25], that is an application of the detailed balance principle. The evaporation probability for a particle of type $j$, mass $m_{j}$, spin $S_{j} \cdot \hbar$, and kinetic energy $E$ is given by

$$
P_{j}(E) d E=\frac{\left(2 S_{j}+1\right) m_{j}}{\pi^{2} \hbar^{3}} \sigma_{\mathrm{inv}} \frac{\rho_{f}\left(U_{f}\right)}{\rho_{i}\left(U_{i}\right)} E d E
$$

where $\rho$ 's are the nuclear level densities $\left(\rho_{f}\left(U_{f}\right)\right.$ for the final nucleus, $\rho_{i}\left(U_{i}\right)$ for the initial one), $U_{i} \equiv U$ is the excitation energy of the evaporating nucleus, $U_{f}=U-E-Q_{j}$ that of the final one, $Q_{j}$ is the reaction $Q$ for emitting a particle of type $j$ from the original compound nucleus, and $\sigma_{\text {inv }}$ is the cross section for the inverse process.

Eq. (11) must be implemented with a suitable form for the nuclear level density and the inverse cross sections. Many recipes have been suggested for both. In the original work of Dostrovsky [26], $\rho(U) \approx C \exp (2 \sqrt{a U})$, with $a=A / 8$ has been used for the level density dependence on the excitation energy $U$. This has led to a simple form for the evaporation probability:

$$
P_{j}(E) d E=\frac{\left(2 S_{j}+1\right) m_{j}}{\pi^{2} \hbar^{3}} \sigma_{\mathrm{inv}} \frac{e^{2 \sqrt{a\left(U-E-Q_{j}\right)}}}{e^{2 \sqrt{a U}}} E d E .
$$

In the same work, the inverse cross sections have been parametrized in a very simple way, so that expression (12) can be analytically integrated and used for MC sampling. The same formulation is used in this work with, however, a different choice of $a$ as it will be discussed later.

The total width for neutron emission can be found by integrating Eq. (11) between zero and the maximum possible ejectile energy $\left(U-Q_{j}\right)$

$$
\Gamma_{j}=\frac{\left(2 S_{j}+1\right) m_{j}}{\pi^{2} \hbar^{2}} \int_{0}^{\left(U-Q_{j}\right)} \sigma_{\mathrm{inv}}(E) \frac{\rho_{f}}{\rho_{i}} E d E .
$$

The same applies to charged particles, where the integration actually goes from some effective Coulomb barrier where $\sigma_{\text {inv }}$ drops to zero, up to the maximum energy.

The evaporative process is in competition with another equilibrium process, that is fission [27]. For the fission probability, a statistical method can be used [25, 28]: obtaining for the total fission width

$$
\Gamma_{\mathrm{F}}=\frac{1}{2 \pi} \frac{1}{\rho_{i}(U)} \int_{0}^{\left(U-B_{\mathrm{F}}\right)} \rho_{\mathrm{F}}\left(U-B_{\mathrm{F}}-E\right) d E
$$

where $B_{\mathrm{F}}$ is the fission barrier, and $\rho_{\mathrm{F}}\left(U_{\mathrm{F}}\right) \approx C \exp \left(2 \sqrt{a_{\mathrm{F}} U_{\mathrm{F}}}\right)$, the level density of the fissioning nucleus at the saddle point, where the excitation energy $U_{\mathrm{F}}$ is given by the initial one minus the fission barrier.

We follow the prescriptions of Atchison [29] to calculate the quantities entering Eq. (14), except, again, for the level density parameter $a_{\mathrm{F}}$. 
In both $\rho_{\mathrm{F}}(U)$ and $\rho(U)$ we use the so-called backshifted level density, using $U-\Delta$ rather than $U$, where $\Delta$ is the pairing energy. Moreover, $\tilde{a}=a / A$, and $\tilde{a}_{\mathrm{F}}=a_{\mathrm{F}} / A$ are found to be all but constant parameters: they possess a dependence on $A$ and $Z$, due to shell and deformation effects, and a dependence on excitation energy. Both effects have been experimentally observed, and have been subject of many phenomenological and theoretical investigations (see [30, 31, 32, 33, 34, 35]). Here the $N$ and $Z$ dependence of Ref. [30] is used, and complemented with the energy dependence prescription of Ignatyuk [31, 32]

$$
\begin{aligned}
a & =A \cdot[\bar{a} \cdot f(U)+\tilde{a} \cdot(1-f(U))] \\
\bar{a} & =a_{0}+9.17 \times 10^{-3} \cdot\left[S_{\mathrm{Z}}(Z)+S_{\mathrm{N}}(N)\right] \\
\tilde{a} & =0.154-6.3 \times 10^{-5} \cdot A \\
f(U) & =\frac{1-e^{-0.054 \cdot(U-\Delta)}}{0.054 \cdot(U-\Delta)}
\end{aligned}
$$

where according to [30, $a_{0}$ is given by $0.142 \mathrm{MeV}^{-1}$ and $0.12 \mathrm{MeV}^{-1}$ for undeformed and deformed nuclei respectively, and $S_{Z}(Z)$ and $S_{N}(N)$ are the shell correction terms for protons and neutrons. The unit of energy used throughout Eq. (15) is $\mathrm{MeV}$.

The level density at the saddle point $\rho_{\mathrm{F}}$ is different from that of the nucleus in its ground state. From comparison to experimental data, it turns out that $a_{\mathrm{F}}$ is greater than the $a$ used for evaporation of about $10 \%$ at low excitation energies, and the two $a$ 's become equal at large excitation energies. We use $a_{\mathrm{F}} \approx 1.08 a$, with a smooth $A$ dependence. After fission occurs, the two fragments are treated like independent residual nuclei with their own excitation and can possibly emit further particles.

For light nuclei, the statistical assumptions and the sequential emission scheme underlying the classical evaporation models become less and less applicable, because:

- Already moderate excitation energies can represent a substantial fraction of the (total) binding energy of such nuclei.

- The level structure of such nuclei is usually highly specific and anyway level spacings can be comparable with the excitation energy.

- The "evaporation" of light fragments other than p or $\mathrm{n}$ becomes meaningless, since the mass of the "evaporated" fragment can be comparable or even larger than the mass of the residual nucleus.

Therefore other deexcitation mechanisms are more suitable for these light residual nuclei. The one adopted for this calculations is the so called Fermi Break-up model [36, 37], where the excited nucleus is supposed to disassemble just in one step into two or more fragments, with branching given by plain phase space considerations. In particular, the probability for disassembling a nucleus of $N$ neutrons, $Z$ protons, and $U$ excitation energy (total mass $\left.M^{*}=U+M_{A, Z}\right)$ into $n$ fragments $(n \geq 2)$ of the same total charge and baryon number, is given by:

$$
W=\frac{g}{G}\left[\frac{V_{\mathrm{br}}}{(2 \pi \hbar)^{3}}\right]^{n-1}\left(\frac{1}{M^{*}} \prod_{i=1}^{n} m_{i}\right)^{3 / 2} \frac{(2 \pi)^{3(n-1) / 2}}{\Gamma\left(\frac{3}{2}(n-1)\right)} E_{\mathrm{kin}}^{3 n / 2-5 / 2}
$$


where the spin factor $g$, and the permutation factor $G$ are given by $\left(n_{j}\right.$ is the number of identical particles of $j$ th kind)

$$
g=\prod_{i=1}^{n}\left(2 S_{i}+1\right), \quad G=\prod_{j=1}^{k} n_{j} !
$$

and $E_{\text {kin }}$ is the total kinetic energy of all fragments at the moment of break-up. $V_{\text {br }}$ is a volume of the order of the initial residual nucleus volume. Therefore, the final state are conveniently selected by means of a MC procedure, by evaluating such an expression for all possible combinations of fragments energetically allowed and making a random selection. We considered all combinations formed by up to six fragments, unless the residual "nucleus" is composed by $A$ like particles ( $\mathrm{p}$ or $\mathrm{n}$ ), in which case it is disintegrated into $A$ fragments according to phase space. All particle stable states with $A \leq 16$ have been included, plus the particle unstable levels with sizeable $\gamma$ decay branching ratios. Also a few known particle unstable isotopes, like ${ }^{8} \mathrm{Be}$, have been included and, if produced, are let to decay according to the experimental branching. Once the final state configuration has been selected, the kinematical quantities of each fragment are chosen according to $n$-body phase space distribution. Such a selection must be performed taking care to subtract from the available energy the Coulomb repulsion of all charged particles: the Coulomb energy is then added back to the charged particles alone, to simulate properly the effect of the Coulomb repulsion. In practice $E_{\text {kin }}$ at disassembling will be given by:

$$
E_{\mathrm{kin}}=U-\left(\sum_{i=1}^{n} m_{i}-M_{A, Z}\right)-B_{\mathrm{Coul}}
$$

where it must be recalled that the emitted fragments can be in an excited state. The total Coulomb barrier $B_{\text {Coul }}$ of the selected configuration is distributed to charged particles after disassembling, in their own c.m. system.

According to the picture of the compound nucleus like an equilibrated system determined only by its mass, charge and excitation energy, with no memory of previous steps of the interaction, Fermi Break-up is activated in the model every time the current compound nucleus has mass number $A \leq 17$, including possible light fission fragments. The fragmentation of higher mass compound nuclei is not yet included in the model. This process, although its cross section is quite small, is important when considering the distribution of residual nuclei, because it can produce isotopes very far both from the target mass and from the fission product distribution.

\section{The production of grey and black particles and resid- ual nuclei in high energy collisions}

\subsection{Grey and black particles and correlations}

The intranuclear cascade of low energy secondaries and the evaporation of nucleons and light fragments mainly contribute to the production of hadrons and light fragments with a velocity less than about $0.7 c$ in the rest frame of the target nucleus. They are frequently called "target 
associated" or "slow" particles. Most of the experimental information on slow particle production presently comes from experiments using nuclear emulsions as targets. The emulsions usually consist of a component of light nuclei (H,C,N,O) and a component of heavy nuclei $(\mathrm{Ag}, \mathrm{Br})$. The appearance of slow particles in these experiments has led to their subdivision into "grey" and "black" particles. The exact definition usually differs slightly between different experiments. As shown by many authors this subdivision has not only an experimental meaning but also subdivides slow particle production into a region which can be understood by intranuclear cascade processes (grey particle production) and a region of black particle production based on nuclear evaporation processes. If not explicitly stated, throughout this paper we apply the following definitions: grey particles are assumed to be singly charged particles with a Lorentz- $\beta$ value between 0.23 and 0.7 and black particles are singly and multiply charged particles with $\beta<0.23$. This is in agreement with definitions usually assumed in experiments 38, 39]. Furthermore, within our calculation we use the emulsion-composition from 38], i.e. an emulsion consisting of $28.8 \%$ of light nuclei and of $71.4 \%$ of heavy nuclei.

In Tab.11 we give the average multiplicities of grey, black, and heavy (=grey+black) particles in proton-emulsion interactions as obtained with our MC model DTUNUC 2.0 together with experimental results for different momenta of the projectile proton. In addition this is shown in Fig. 1 together with the corresponding shower particle $(\beta>0.7)$ multiplicities. Whereas the average number of shower particles is increasing throughout the whole energy range we get an increasing multiplicity of grey and black particles up to about $40 \mathrm{GeV} / c$ which turns into an almost constant behaviour for higher energies. Within our model, this constant behaviour is due to limiting fragmentation in each hadron-nucleon interaction [24] together with a constant formation zone intranuclear cascade and inelastic hadron-nucleus cross sections depending only weakly on the projectile energy. The position in energy of the threshold region, i.e. the region of increasing heavy particle multiplicities, is governed by the nuclear geometry and the nuclear potential, which both are treated in a very rough manner, and by the way the Glauber cascade is biased by lower cuts applied to chain masses. Further shifts of this region or changes of the slope in the threshold region can be obtained by varying the distributions from which the x-values of the sea-partons are sampled". The experimental data are taken from a compilation of data by Fredriksson et al. [40], i.e. they were obtained in different experiments. The definitions of "grey" and "black" may therefore slightly vary between them. This fact may also partly account for the fluctuations within the data for grey and black particle multiplicities in the high energy region. For momenta above about $20 \mathrm{GeV} / c$ the model agrees well with measured multiplicities, whereas at low energies our results seem to depart from the experiments. However, the different definitions used for "grey" and "black" cannot completely explain the big differences within the experimental results at low energies and any clear experimental information on the threshold behaviour is missing. From this comparison it is therefore difficult to draw conclusions about the applicability of our model of slow particle production to energies below 10-20 GeV and to modifications within the model which could be necessary to reproduce the observed threshold behaviour.

In order to investigate how the two emulsion components contribute to the energy dependence of the average multiplicities discussed so far we show in Tabs.2 and 3 and, together with

\footnotetext{
${ }^{4}$ Within DTUNUC they are sampled from a $1 / x$-distribution $[$.
} 
shower particle multiplicities, in Fig.5 the mean grey, black, and heavy particle multiplicities for the light and heavy component separately in comparison to experimental data [40]. In order to illustrate the uncertainties within the experimental data we give for some energies several multiplicity values, which were measured in different experiments. As it is clearly shown in Fig.5 5 a in interactions of protons with light nuclei even at high energies only up to three target nucleons are interacting with the projectile, i.e. we are dealing with a very limited Glauber cascade and, therefore, do not observe the typical breakdown of the cascade at low energies which would manifest itself in decreasing grey and black particle multiplicities. In agreement with the measurements we obtain constant mean grey and black particle multiplicities in the whole energy range. It seems, that the model overestimates the black particle multiplicity which could be due to the absence of the treatment of the nuclear skin in the nuclear potential, i.e. by underestimating the low part of the excitation energy distribution. However we must note, that the experiments were usually classifying the target nuclei as belonging to one of the components by the number of produced heavy particles, which implies further uncertainties. An average value of one grey particle per interaction agrees well with the experimental results. The model reproduces the measured multiplicities of slow particles in interactions of protons with nuclei of the heavy component (Tab.3, Fig.5b) down to a proton momentum of about $20 \mathrm{GeV} / c$.

In Figs.6 and 7 we present the grey and black particle multiplicity distributions normalized to unity for proton-emulsion interactions at $200 \mathrm{GeV}$ (a) and $\Sigma^{-}$-emulsion interactions at $350 \mathrm{GeV}$ (b) together with data [38, 41]. As the comparisons show, our model is able to reproduce the data on slow particle multiplicities very well. The grey particle multiplicity distribution for proton projectiles (Fig.6a) slightly underestimates the measured distribution at high multiplicities which, however, might be not very conclusive since the uncertainties within the experimental data are rather big in this region. The hump in the calculated black particle distribution for proton projectiles at $N_{b} \approx 4$ (Fig. (7a) is due to the evaporation of charged particles from light emulsion nuclei and seems to be less pronounced in the measured distribution. This is not the case in the $\Sigma^{-}$-emulsion data (Fig.7 $\mathrm{b}$ ), where, on the other hand, the uncertainties are higher than within the proton-emulsion data.

The reasonable description of slow particle multiplicity distributions implies that the model should be able to reproduce measured correlations between grey, black, and shower particle multiplicities. In Fig.8 we compare correlations between grey and shower particle multiplicities $(\mathrm{a}, \mathrm{b})$ and between black and shower particle multiplicities (c,d) and in Fig.9a,b between grey and black particle multiplicities with data of the Alma-Ata-Leningrad-Moscow-Tashkent Collab. [39] on proton-emulsion interactions at $200 \mathrm{GeV}$ and in Fig.9b in addition to data of the KLM-Collab. [42]. In Ref. [39] the errorbars are obviously only given for selected data points. Apart from the correlation between grey and black particles, where we obtain slightly more black particles for a fixed number of grey particles than seen in the experiments, our calculations are in good agreement with the data within their uncertainties.

A detailed experimental study of slow particle production in interactions of protons, pions, and kaons with different target nuclei at energies varying between $50 \mathrm{GeV}$ and $150 \mathrm{GeV}$ was presented in 43. Here, grey particles are defined as charged particles having a velocity between $0.3 c$ and $0.7 c$. In Tab. to these data. Again, the agreement is satisfactorily. 
The dependence of the mean grey, heavy, and shower particle multiplicities on the mass number of the target nucleus in proton-nucleus collisions at $300 \mathrm{GeV} / c$ was subject to further comparisons. The results are given in Tab.5 and Fig.10 together with data taken from Ref. 40]. Our model is reproducing the increase of the heavy particle multiplicity with the target mass number. As mentioned above the data point for $\mathrm{C}, \mathrm{N}, \mathrm{O}$ was obtained in emulsion experiments in which the identities of the target nuclei were deduced from the heavy particle multiplicities. Therefore this data point has to be taken with care.

Finally, we compare grey particle multiplicity distributions in interactions of protons and pions with different target nuclei at $200 \mathrm{GeV} / c$ with recent data of the WA80-Collab. [44]. In agreement with the experiment grey particles are defined as singly charged particles with a kinetic energy between $30 \mathrm{MeV}$ and $400 \mathrm{MeV}$ emitted in the target rapidity region $(-1.7<$ $\eta<1.3$ ). The result of the comparison is shown in Fig.11. All calculated distributions are normalized to the Glauber cross sections of the corresponding interactions. For the two light nuclei $(\mathrm{C}, \mathrm{Al})$ our distributions are consistently broader than the experimental distributions, whereas for heavier targets we agree well in shape and absolute normalization with the data.

\subsection{Residual nuclei and high energy fission}

After evaporation most of the residual nuclei have lost up to one-third of their nucleons depending on their mass $A_{T}$, on the kind and energy of the projectile, and on the interaction characteristics (impact parameter etc.). They may be considered as heavy fragments produced in a spallation or a deep spallation process. In addition, the high energy fission model and the Fermi Break-up model which were introduced in Sect.3 modify the mass spectrum of the nuclear prefragments furthermore. In Fig.12 we show the isobaric mass yields of fragments in interactions of silver nuclei with $11.5 \mathrm{GeV}$ (a) and $300 \mathrm{GeV}$ (b) protons together with data [45, 46]. Since multifragmentation is not included in our MC model we get - apart from light fragments $(A \leq 4)$ which were evaporated from the residual nucleus - almost no fragments with masses below $A_{\text {res }} \approx 40$. In the spallation region $\left(50 \leq A_{\text {res }} \leq 100\right)$ our calculation agrees within a factor of two with the measured mass yields, which is satisfactorily in view of our simplified approach and taking into account the fact that multifragmentation would lead to a further decrease of the cross section. The rising yields of fragments close to the target mass $\left(A_{T}-5 \leq A_{\text {res }} \leq A_{T}\right)$ are not described within our model. This is due to the fact that such processes like quasi-elastic scattering are not treated within our model and it might be due to our rough treatment of the nuclear potential, i.e. we probably underestimate the low part of the excitation energy distribution by neglecting the nuclear skin effects. As experimental results on isotope production show, fragment production cross sections remain about constant for projectile energies above $10 \mathrm{GeV}$ (see Fig.12 and 477, 48). This fact suggests that the regime of constant slow particle production may already be reached at an energy of about $10 \mathrm{GeV}$. In contrast, within our model the threshold above which slow particle production does not change significantly is at about 20-30 GeV (cf. discussion in Sect.4.1). This fact explains the different shape of the calculated mass yields at $E_{\mathrm{Lab}}=11.5 \mathrm{GeV}$ (Fig.12a) as compared to $E_{\mathrm{Lab}}=300 \mathrm{GeV}$ (Fig.12]b). However, in order to draw further conclusions on the threshold region which are based on fragment production cross sections, it would be necessary to describe all aspects of the fragmentation process (such as multifragmentation) which is 
beyond the scope of this work.

In Fig.13 we compare the charge yield obtained in interactions of $10.6 \mathrm{GeV}$ protons on ${ }^{197} \mathrm{Au}$ nuclei (a) and the isobaric mass yield obtained in interactions of $800 \mathrm{GeV}$ protons on ${ }^{197} \mathrm{Au}$ nuclei (b) to data [49, 50]. Since high energy fission significantly modifies the fragment production cross sections we show both, the mass yields of the residual nuclei after the evaporation-step without high energy fission (crosses) and mass yields obtained taking high energy fission into account (diamonds). Within the limitations of our models we are able to reproduce the measured yields very well, especially the yields at $E_{\mathrm{Lab}}=800 \mathrm{GeV}$ (Fig.13b) where we agree with the data in the mass range $60 \leq A_{\text {res }} \leq 190$ within their uncertainties. The calculated yields at $A_{\text {res }}=2,3,4$ represent light fragments evaporated from the prefragments. Again, our models do not cover the multifragmentation region and the mass region very close to the target mass.

In order to investigate isotope-production in more detail we compare independent mass yield distributions from interactions of $800 \mathrm{GeV}$ protons with ${ }^{197} \mathrm{Au}$ with data [50] in Fig. 14. There we plot the cross sections for the production of certain isotopes with masses $A_{\text {res }}$ and charge $Z$ versus the difference of their charge and the most probable charge $Z_{\mathrm{mp}}$ for three intervals of $A_{\text {res }}$. Corresponding to [50] $Z_{\mathrm{mp}}$ is defined as

$$
Z_{\mathrm{mp}}(A)=a A^{2}+b A+c
$$

with $a=-0.382 \cdot 10^{-3}, b=0.483, c=0$ for $82 \leq A_{\text {res }} \leq 89$, and $c=0.231$ for $122 \leq A_{\text {res }} \leq 129$. In the highest mass range $166 \leq A_{\text {res }} \leq 176 c$ had to be modified by 1.0 in order to compare the shape of the distributions, i.e. $c=1.254$. For each interval we calculate the independent yields for three different $A_{\text {res }}$ values. We are able to reproduce the measured charge distributions which have the typical gaussian shape.

The average recoil momenta of the fragments in proton- ${ }^{197} \mathrm{Au}$ interactions at $800 \mathrm{GeV}$ as a function of the mass loss $\Delta A=A_{T}-A_{\text {res }}$ are shown together with data from different experiments [51] in Fig.15. The momenta of the fragments obtained with our MC model are in reasonable agreement with the data.

\section{$5 \quad$ Summary and conclusions}

We have extended Monte Carlo models based on the Dual Parton Model for high energy hadron-nucleus collisions to the calculation of cross sections for residual nuclei production and to nuclear evaporation, Fermi Break-up, and high energy fission.

As it has been demonstrated in a number of past studies [2, 3, 4, 5] the models used agree quite well with momentum distributions and multiplicities of hadrons produced in high energy interactions. Here we find in addition a quite good agreement of the average numbers of grey prongs $\left\langle N_{g}\right\rangle$ and black prongs $\left\langle N_{b}\right\rangle$ as function of the collision energy and as function of the target nucleus with experimental data, which were mostly obtained in emulsion experiments.

Furthermore, calculated multiplicity distributions of grey and black prongs agree well to data. The correlations between the fast shower particles and grey and black prongs as well as the correlations between grey and black prongs are often used to analyze the observed events 
in terms of centrality of the collision or in terms if the impact parameter. Our Monte Carlo events show all of these correlations in good agreement with experimental results.

We find a reasonable agreement of the calculated cross-sections with data for the production of residual nuclei in most of the mass-region below the mass of the original target nucleus. Since our model is formulated only in terms of average nuclear properties we can not reproduce all the fluctuations, which are due to particular properties of individual nuclei.

At high energies we find the average numbers of grey and black prongs to become independent from the collision energy. This is a behaviour which can be traced back to the limiting fragmentation property of hadron-hadron collisions in the target or projectile rest frame. The threshold region, where this high energy behaviour is reached is difficult to predict in a model like ours. As the model has enough freedom to adjust the threshold behaviour to the behaviour of the data, a further tuning of the model parameters might be possible as soon as more consistent data become available.

\section{Acknowledgements}

One of the authors (S.R.) acknowledges stimulating discussions with F.W. Bopp and W. Heinrich. 


\section{References}

[1] A. Capella, U. Sukhatme, C. I. Tan and J. Tran Thanh Van: Phys. Rep. 236 (1994) 227

[2] P. Aurenche, F. W. Bopp, A. Capella, J. Kwiecinski, M. Maire, J. Ranft and J. Tran Thanh Van: Phys. Rev. D45 (1992) 92

[3] F. W. Bopp, R. Engel, D. Pertermann and Ranft: Phys. Rev. D49 (1994) 3236

[4] H.-J. Möhring and J. Ranft: Z. Phys. C52 (1991) 643

[5] J. Ranft: Phys. Rev. D51 (1995) 64

[6] A. Fassò, A. Ferrari, J. Ranft and P. R. Sala: FLUKA: present status and future developments, in Proceedings of the IVth International Conference on Calorimetry in High Energy Physics, La Biodola (Elba), Italy, 1993, ed. by A. Menzione and A. Scribano, World Scientific, p. 493, 1994

[7] A. Fassò, A. Ferrari, J. Ranft and P. R. Sala: FLUKA: Performances and Applications in the Intermediate Energy Range, in Proceedings of the Specialists Meeting on Shielding Aspects of Accelerators, Targets \& Irradiation Facilities, Arlington, U.S.A., 1994, published by OECD/NEA, p. 287, 1994

[8] P. A. Aarnio et al.: in Proceedings of the International Conference on Monte Carlo Simulation in High Energy and Nuclear Physics, MC'93, Tallahassee, U.S.A., 1993, ed. by P. Dragovitsch, S. L. Linn and M. Burbank, World Scientific, p. 88, 1994

[9] J. Ranft and J. T. Routti: Particle Accelerators 4 (1972) 101

[10] H. W. Bertini: Phys. Rev. 137 (1963) 1801

[11] H. W. Bertini: Phys. Rev. 188 (1969) 1711

[12] Y. Yariv and Z. Fraenkel: Phys. Rev. C24 (1981) 488

[13] L. Stodolski: Proc. Vth Int. Colloquium on Multiparticle Reactions, Oxford (1975) 577

[14] J. Ranft: Z. Phys. C43 (1989) 439

[15] J. Ranft: Phys. Rev. D37 (1988) 1842

[16] R. Engel: Z. Phys. C66 (1995) 203

[17] S. Y. Shmakov, V. V. Uzhinskii and A. M. Zadoroshny: Comput. Phys. Commun. 54 (1989) 125

[18] P. Aurenche, F. W. Bopp, R. Engel, D. Pertermann, J. Ranft and S. Roesler: Comput. Phys. Commun. 83 (1994) 107

[19] T. Sjöstrand: Comput. Phys. Commun. 39 (1986) 347 
[20] T. Sjöstrand and M. Bengtsson: Comput. Phys. Commun. 43 (1987) 367

[21] L. Landau and I. Pomeranchuk: Dokl. Akad. Nauk SSR 92 (1953) 535,734

[22] A. Bialas: Z. Phys. C26 (1984) 301

[23] K. Hänssgen and J. Ranft: Comput. Phys. Commun. 39 (1986) 37

[24] J. Benecke, T. T. Chou, C. N. Yang and E. Yen: Phys. Rev. 188 (1969) 2159

[25] V. F. Weisskopf: Phys. Rev. 52 (1937) 295

[26] I. Dostrovsky, Z. Fraenkel and G. Friedlander: Phys. Rev. 116 (1959) 683

[27] R. Vandenbosh and J. R. Huizenga: Nuclear Fission Academic Press New York 1973

[28] N. Bohr and J. A. Wheeler: Phys. Rev. 56 (1939) 426

[29] F. Atchison: Jül-conf-34, Talk given at the Meeting on Targets for neutron beam spallation sources, ed. by G. Bauer, KFA Jülich, Germany, 1980

[30] A. Gilbert and A. G. W. Cameron: Can. J. Phys. 43 (1965) 1446

[31] A. V. Ignatyuk, G. N. Smirenkin and A. S. Tishin: Sov. J. Nucl. Phys. 21 (1975) 255

[32] A. V. Ignatyuk et al.: Sov. J. Nucl. Phys. 21 (1975) 612

[33] S. G. Mashnik: Acta Phys. Slov. 43 (1993) 86

[34] A. S. Iljinov and M. V. Mebel: Nucl. Phys. A543 (1992) 517

[35] S. Shlomo: Nucl. Phys. A539 (1992) 17

[36] E. Fermi: Prog. Theor. Phys. 5 (1950) 1570

[37] M. Èpherre and È. Gradsztajn: J. Physique 18 (1967) 48

[38] E. Stenlund and I. Otterlund: Nucl. Phys. B198 (1982) 407

[39] Alma-Ata-Leningrad-Moscow-Tashkent Collab.: Sov. J. Nucl. Phys. 22 (1975) 380

[40] S. Fredriksson, G. Eilam, G. Berlad and L. Bergström: Phys. Rep. 144 (1987) 187 and references therein

[41] M. Szarska et al.: Phys. Rev. D47 (1993) 784

[42] KLM Collab.: A. Dabrowska et al.: Z. Phys. C59 (1993) 399

[43] K. Braune et al.: Z. Phys. C13 (1982) 191

[44] WA80 Collab.: R. Albrecht et al.: Z. Phys. C57 (1993) 37 
[45] G. English, N. T. Porile and E. P. Steinberg: Phys. Rev. C10 (1974) 2268

[46] N. T. Porile, G. T. Cole and C. Rudy: Phys. Rev. C19 (1979) 2288

[47] S. B. Kaufman et al.: Phys. Rev. C14 (1976) 1121

[48] J. Hüfner: Phys. Rep. 125 (1985) 129

[49] W. Heinrich et al.: Radiation Measurements 25 (1995) 203

[50] L. Sihver et al.: Nucl. Phys. A543 (1992) 703

[51] D. J. Morrissey: Phys. Rev. C39 (1989) 460 


\section{Tables}

Table 1: Multiplicities of grey $\left(N_{g}\right)$, black $\left(N_{b}\right)$, and heavy $\left(N_{h}=N_{g}+N_{b}\right)$ particles in interactions of protons with emulsion nuclei are given for different momenta of the incident proton. The values as obtained with our model DTUNUC are compared to data from various experiments [40]. Within our results we define "grey particles" as particles with a velocity $\beta=$ $v / c$ between 0.23 and 0.7 and therefore "black particles" as particles with $\beta<0.23$. Within the experimental data an upper $\beta$-limit for grey particles of 0.7 is usually assumed, whereas the $\beta$-cut between "grey" and "black" may slightly differ between different experiments.

\begin{tabular}{|r||c|c|c|c|c|c|}
\hline \multicolumn{1}{|r||}{$p_{\text {Lab }}$} & \multicolumn{2}{|c|}{$\left\langle N_{g}\right\rangle$} & \multicolumn{2}{c|}{$\left\langle N_{b}\right\rangle$} & \multicolumn{2}{c|}{$\left\langle N_{h}\right\rangle$} \\
$(\mathrm{GeV} / c)$ & DTUNUC & Exp. & DTUNUC & Exp. & DTUNUC & Exp. \\
\hline \hline 6.2 & 2.0 & $3.58 \pm 0.11$ & 3.2 & & 5.2 & $9.25 \pm 0.18$ \\
\hline 9.0 & 2.3 & $3.1 \pm 0.4$ & 3.6 & $4.7 \pm 0.5$ & 5.9 & \\
\hline 14.9 & 2.4 & & 4.2 & & 6.6 & 8.4 \\
\hline 21.0 & 2.6 & $2.9 \pm 0.2$ & 4.6 & $4.6 \pm 0.2$ & 7.2 & \\
\hline 24.0 & 2.5 & $3.17 \pm 0.1$ & 4.5 & & 7.0 & $7.7 \pm 0.2$ \\
\hline 50.0 & 2.8 & $3.07 \pm 0.1$ & 5.0 & & 7.8 & $7.5 \pm 0.2$ \\
\hline 67.0 & 2.9 & $2.5 \pm 0.1$ & 5.2 & $4.7 \pm 0.2$ & 8.1 & \\
\hline 67.0 & & $2.85 \pm 0.09$ & & & & $7.5 \pm 0.2$ \\
\hline 200.0 & 2.9 & $2.48 \pm 0.08$ & 5.4 & $4.79 \pm 0.12$ & 8.3 & \\
\hline 300.0 & 2.9 & $2.6 \pm 0.2$ & 5.4 & 5.4 & 8.3 & $7.1 \pm 0.2$ \\
\hline 400.0 & 2.9 & & 5.4 & & 8.3 & $8.1 \pm 0.2$ \\
\hline
\end{tabular}


Table 2: Grey $\left(N_{g}\right)$, black $\left(N_{b}\right)$, and heavy $\left(N_{h}=N_{g}+N_{b}\right)$ particle multiplicities in interactions of protons with light emulsion nuclei $(\mathrm{C}, \mathrm{N}, \mathrm{O})$ are given for different momenta of the incident proton. Results of the model are compared to data from various experiments 40]. For the definition of grey and black particles we refer to the caption of Tab. [1.

\begin{tabular}{|r||c|c|c|c|c|c|}
\hline \multicolumn{1}{|c||}{$p_{\text {Lab }}$} & \multicolumn{2}{c|}{$\left\langle N_{g}\right\rangle$} & \multicolumn{2}{c|}{$\left\langle N_{b}\right\rangle$} & \multicolumn{2}{c|}{$\left\langle N_{h}\right\rangle$} \\
$(\mathrm{GeV} / c)$ & DTUNUC & Exp. & DTUNUC & Exp. & DTUNUC & Exp. \\
\hline \hline 6.0 & 0.9 & $2.54 \pm 0.06$ & 3.2 & & 4.1 & $8.05 \pm 0.1$ \\
\hline 6.0 & & $0.96 \pm 0.07$ & & & & $2.67 \pm 0.14$ \\
\hline 9.0 & 0.86 & $1.4 \pm 0.1$ & 3.1 & & 3.96 & $4.7 \pm 0.1$ \\
\hline 21.0 & 1.02 & $0.7 \pm 0.1$ & 2.9 & $2.2 \pm 0.1$ & 3.92 & $2.9 \pm 0.1$ \\
\hline 26.0 & 1.05 & $0.91 \pm 0.04$ & 2.9 & & 3.95 & $2.5 \pm 0.1$ \\
\hline 50.0 & 1.03 & $0.91 \pm 0.04$ & 2.8 & & 3.83 & $2.4 \pm 0.1$ \\
\hline 60.0 & 1.07 & $0.63 \pm 0.07$ & 2.9 & $2.0 \pm 0.2$ & 3.97 & $2.6 \pm 0.2$ \\
\hline 67.0 & & $0.6 \pm 0.1$ & & $1.5 \pm 0.1$ & & \\
\hline 69.0 & 1.05 & $0.84 \pm 0.04$ & 2.8 & & 3.85 & $3.47 \pm 0.15$ \\
\hline 69.0 & & $0.91 \pm 0.05$ & & & & $3.65 \pm 0.1$ \\
\hline 200.0 & 1.06 & $0.9 \pm 0.05$ & 2.8 & $1.8 \pm 0.08$ & 3.86 & $2.7 \pm 0.11$ \\
\hline 200.0 & & & & & & $2.61 \pm 0.08$ \\
\hline 200.0 & & & & & & $2.75 \pm 0.1$ \\
\hline 300.0 & 1.08 & & 2.8 & & 3.88 & $2.9 \pm 0.21$ \\
\hline 400.0 & 1.04 & $0.67 \pm 0.04$ & 2.8 & & 3.84 & $2.47 \pm 0.09$ \\
\hline
\end{tabular}


Table 3: Grey $\left(N_{g}\right)$, black $\left(N_{b}\right)$, and heavy $\left(N_{h}=N_{g}+N_{b}\right)$ particle multiplicities in interactions of protons with heavy emulsion nuclei $(\mathrm{Ag}, \mathrm{Br})$ are given for different momenta of the incident proton. Results of the model are compared to data from various experiments [40. For the definition of grey and black particles we refer to the caption of Tab. 1.

\begin{tabular}{|c|c|c|c|c|c|c|}
\hline \multirow{2}{*}{$\begin{array}{r}p_{\text {Lab }} \\
(\mathrm{GeV} / c)\end{array}$} & \multicolumn{2}{|c|}{$\left\langle N_{g}\right\rangle$} & \multicolumn{2}{|c|}{$\left\langle N_{b}\right\rangle$} & \multicolumn{2}{|c|}{$\left\langle N_{h}\right\rangle$} \\
\hline & DTUNUC & Exp. & DTUNUC & Exp. & DTUNUC & Exp. \\
\hline 9.0 & 2.8 & $4.1 \pm 0.5$ & 4.0 & & 6.8 & $10.2 \pm 0.8$ \\
\hline 13.8 & 2.9 & $6.6 \pm 0.6$ & 4.7 & & 7.6 & $16.0 \pm 1.4$ \\
\hline 21.0 & 3.1 & $3.9 \pm 0.2$ & 5.3 & $5.9 \pm 0.3$ & 8.4 & \\
\hline & & $3.9 \pm 0.2$ & & & & $9.8 \pm 0.3$ \\
\hline 24.0 & 3.2 & $5.1 \pm 0.6$ & 5.4 & & 8.6 & $16.0 \pm 1.5$ \\
\hline 24.0 & & $3.96 \pm 0.13$ & & & & $9.5 \pm 0.3$ \\
\hline 26.0 & 3.2 & $3.3 \pm 0.1$ & 5.4 & & 8.6 & $11.2 \pm 0.15$ \\
\hline 50.0 & 3.5 & $3.86 \pm 0.13$ & 6.0 & & 9.5 & $9.4 \pm 0.3$ \\
\hline 60.0 & 3.6 & $3.4 \pm 0.2$ & 6.3 & $4.9 \pm 0.6$ & 9.7 & $8.3 \pm 0.6$ \\
\hline 67.0 & 3.5 & $3.4 \pm 0.2$ & 6.1 & $6.2 \pm 0.3$ & 9.5 & \\
\hline 67.0 & & $3.1 \pm 0.1$ & & & & $9.7 \pm 0.3$ \\
\hline 200.0 & 3.8 & $3.29 \pm 0.1$ & 6.6 & $6.36 \pm 0.16$ & 10.4 & $9.66 \pm 0.24$ \\
\hline 200.0 & & & & & & $9.92 \pm 0.17$ \\
\hline 300.0 & 3.9 & & 6.8 & & 10.7 & $9.9 \pm 0.5$ \\
\hline 400.0 & 3.9 & & 6.9 & & 10.8 & $12.4 \pm 0.9$ \\
\hline 400.0 & & $3.8 \pm 0.1$ & & & & $9.9 \pm 0.2$ \\
\hline
\end{tabular}


Table 4: Average grey particle multiplicity for proton, pion, and kaon interactions with nuclei at different energies. The data are from [43]. Grey particles are defined as charged particles with a velocity $v=\beta c$ between $0.3 c$ and $0.7 c$.

\begin{tabular}{|c||c|c|c|c|c|c|}
\hline \multicolumn{1}{|c||}{} & \multicolumn{2}{c|}{$E_{\mathrm{Lab}}=50 \mathrm{GeV}$} & \multicolumn{2}{c|}{$E_{\mathrm{Lab}}=100 \mathrm{GeV}$} & \multicolumn{2}{c|}{$E_{\mathrm{Lab}}=150 \mathrm{GeV}$} \\
& DTUNUC & Exp. & DTUNUC & Exp. & DTUNUC & Exp. \\
\hline \hline $\mathrm{p}-\mathrm{C}$ & 0.74 & $0.91 \pm 0.05$ & 0.73 & $0.82 \pm 0.04$ & 0.77 & $0.89 \pm 0.04$ \\
\hline $\mathrm{p}-\mathrm{Cu}$ & 2.2 & $2.23 \pm 0.11$ & 2.2 & $2.26 \pm 0.11$ & 2.3 & $2.27 \pm 0.11$ \\
\hline $\mathrm{p}-\mathrm{Pb}$ & 4.6 & $4.04 \pm 0.2$ & & & 4.5 & $3.75 \pm 0.19$ \\
\hline$\pi^{+}-\mathrm{C}$ & 0.69 & $0.85 \pm 0.04$ & 0.69 & $0.81 \pm 0.04$ & 0.64 & $0.84 \pm 0.04$ \\
\hline$\pi^{+}-\mathrm{Cu}$ & 1.96 & $1.99 \pm 0.1$ & 1.98 & $2.04 \pm 0.1$ & 1.97 & $1.99 \pm 0.1$ \\
\hline$\pi^{+}-\mathrm{Pb}$ & 4.0 & $3.42 \pm 0.17$ & 3.9 & $2.89 \pm 0.14$ & 3.9 & $3.31 \pm 0.17$ \\
\hline$K^{+}-\mathrm{C}$ & 0.65 & $0.81 \pm 0.04$ & & & 0.67 & $0.80 \pm 0.04$ \\
\hline$K^{+}-\mathrm{Cu}$ & 1.82 & $1.92 \pm 0.1$ & & & 1.76 & $1.93 \pm 0.1$ \\
\hline$K^{+}-\mathrm{Pb}$ & 3.62 & $3.43 \pm 0.17$ & & & 3.77 & $3.23 \pm 0.16$ \\
\hline
\end{tabular}


Table 5: Dependence of the average multiplicities of grey $\left(N_{g}\right)$ and heavy $\left(N_{h}\right)$ particles on the target mass number in proton-nucleus interactions at $p_{\mathrm{Lab}}=300 \mathrm{GeV} / c$. Results of the model are compared to data from various experiments [40]. "Grey" and "heavy" are defined as given in the caption of Tab. 1

\begin{tabular}{|c||c|c|c|c|}
\hline \multicolumn{1}{|c||}{ Nucleus } & \multicolumn{2}{c|}{$\left\langle N_{g}\right\rangle$} & \multicolumn{2}{c|}{$\left\langle N_{h}\right\rangle$} \\
& DTUNUC & Exp. & DTUNUC & Exp. \\
\hline \hline $\mathrm{C}, \mathrm{N}, \mathrm{O}$ & 1.1 & & 3.9 & $2.9 \pm 0.21$ \\
\hline $\mathrm{Al}$ & 1.4 & & 5.4 & \\
\hline $\mathrm{Cr}$ & 2.1 & & 7.3 & $7.2 \pm 0.7$ \\
\hline Emulsion & 2.6 & $2.6 \pm 0.2$ & 7.9 & $7.1 \pm 0.2$ \\
\hline $\mathrm{Ag}, \mathrm{Br}$ & 3.2 & & 9.7 & $9.9 \pm 0.5$ \\
\hline $\mathrm{W}$ & 5.0 & & 13.5 & $12.9 \pm 1.2$ \\
\hline $\mathrm{Au}$ & 5.4 & & 14.2 & \\
\hline
\end{tabular}




\section{Figure Captions}

1. Average excitation energies of residual target nuclei in proton-nucleus interactions before evaporation are shown for different momenta of the incident proton (a). In (b) the average excitation energies are given per nucleon of the residual target nucleus.

2. Target mass dependence of the average excitation energies for residual target nuclei with mass $A_{\text {res }}$ in proton-nucleus interactions before evaporation. $\Delta A$ is the number of nucleons lost by the target nucleus in the primary collision $\left(\Delta A=A_{t}-A_{\text {res }}\right)$.

3. In a) the distribution of excitation energies of gold prefragments in proton-gold interactions at $300 \mathrm{GeV} / c$ is shown for different ranges of prefragment mass $A_{\text {res }}$. The corresponding excitation energy distributions per nucleon of the gold prefragment are given in b).

4. Mean multiplicities of shower, grey, and heavy particles in collisions of protons with emulsion nuclei. Data from various experiments 40 (points) are compared to results of the model (lines).

5. Mean multiplicities of shower, grey, and heavy particles in collisions of protons with emulsion nuclei are shown for the component consisting of light nuclei (C,N,O) (a) and the heavy component (Ag, Br) (b). Data from various experiments 440 (points) are compared to results of the model (lines).

6. Grey particle multiplicity distributions for interactions of protons (a) and $\Sigma^{-}$-hyperons (b) with emulsion nuclei are plotted together with experimental results [38, 41].

7. Black particle multiplicity distributions for interactions of protons (a) and $\Sigma^{-}$-hyperons (b) with emulsion nuclei are plotted together with experimental results [38, 41].

8. The correlations between grey $\left(N_{g}\right)$ and shower $\left(N_{s}\right)$ particle multiplicities (a,b) and black $\left(N_{b}\right)$ and shower particle multiplicities $(\mathrm{c}, \mathrm{d})$ in interactions of protons with emulsion nuclei are compared to experimental results [39.

9. The correlations between grey $\left(N_{g}\right)$ and black $\left(N_{b}\right)$ particle multiplicities in interactions of protons with emulsion nuclei are compared to experimental results [39, 42].

10. Target mass number dependence of the average multiplicities of shower, grey, and heavy particles in proton-nucleus interactions. Data from various experiments [40] (points) are compared to results of the model (lines).

11. The distributions of grey particle multiplicities in proton-nucleus (a) and pion-nucleus (b) interactions as calculated with DTUNUC are compared to experimental results of the WA80-Collab. [44].

12. Mass distributions of prefragments produced in proton-silver interactions at $11.5 \mathrm{GeV}$ (a) and at $300 \mathrm{GeV}$ (b) as obtained with the model are compared to experimental results 45, 46. 
13. Charge distributions of prefragments produced in proton-gold interactions at $10.6 \mathrm{GeV}$ (a) and mass distributions of prefragments produced in proton-gold interactions at $800 \mathrm{GeV}$ (b) as obtained with the model are compared to experimental results [49, 50]. In addition, the distributions obtained without high-energy fission are shown (crosses).

14. The yield distributions from interactions of protons with ${ }^{197} \mathrm{Au}$ nuclei at $800 \mathrm{GeV}$ are shown together with experimental results of Sihver et al. [50] for three different intervals of the mass of the prefragment.

15. The total momentum of residual nuclei as a function of the mass loss of the target nucleus are compared to experimental results. The experimental data are from different experiments and have been taken from Fig. 7 in [50]. 


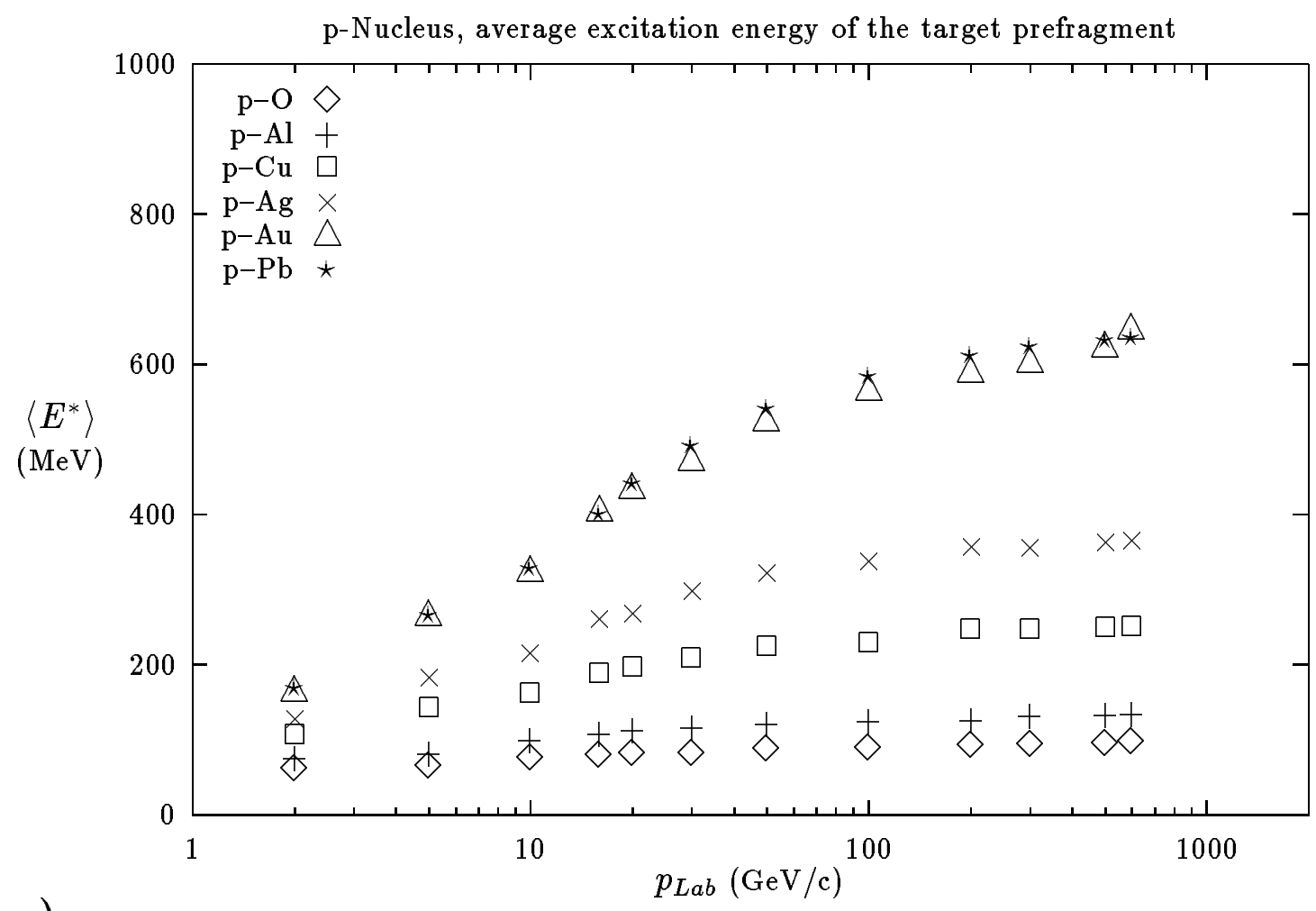

a)

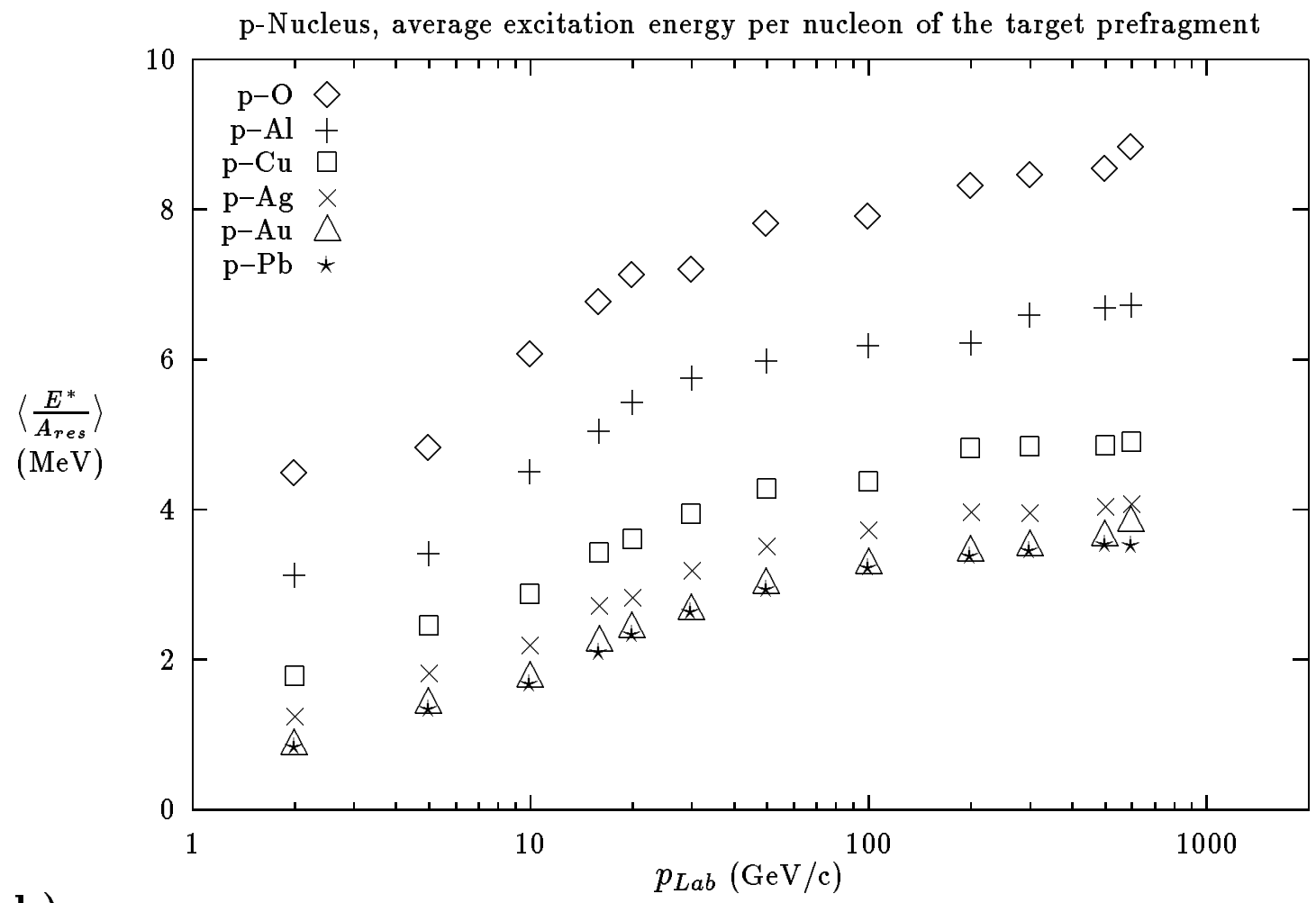

b)

Fig. 1 


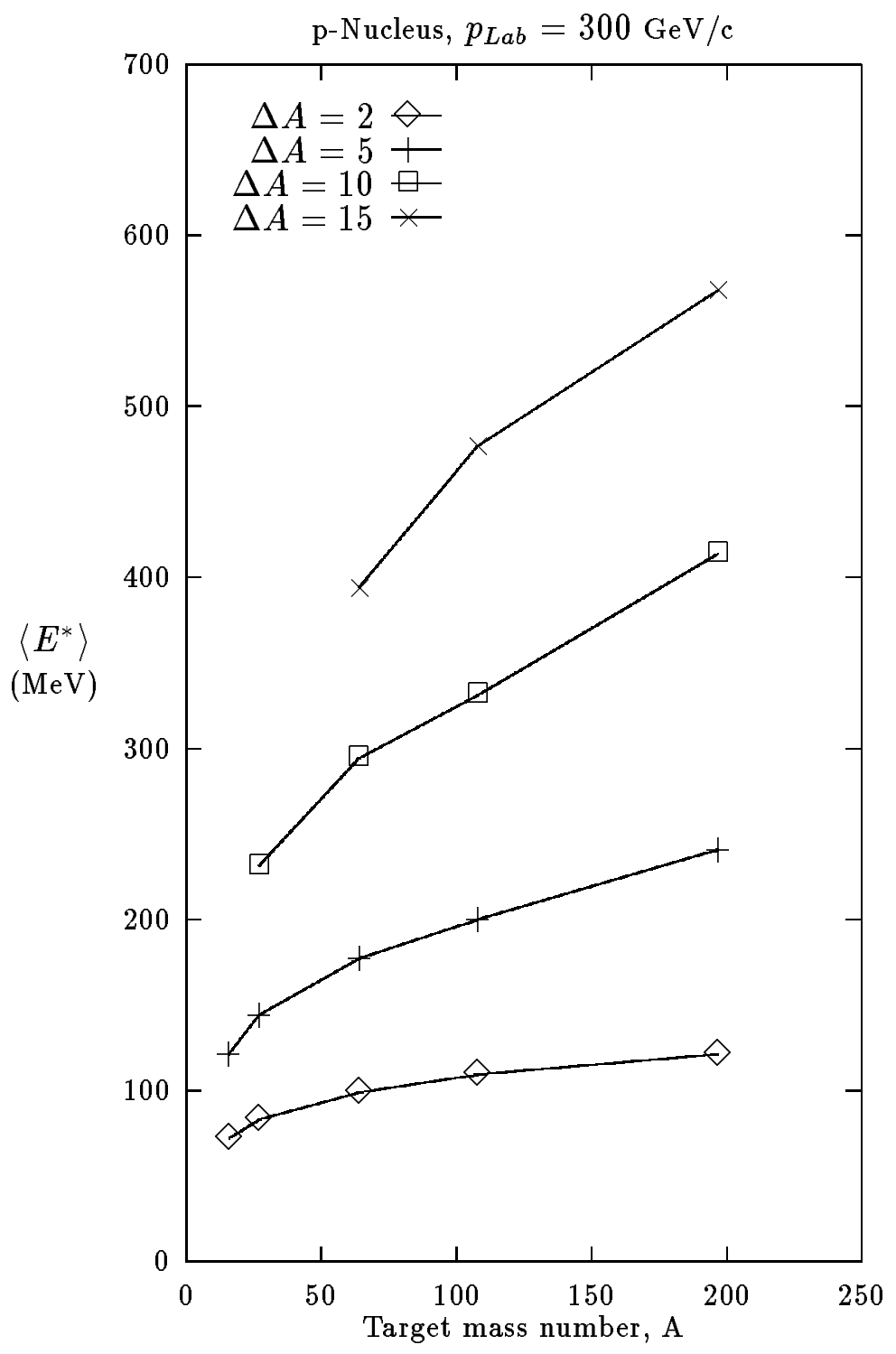

Fig. 2 


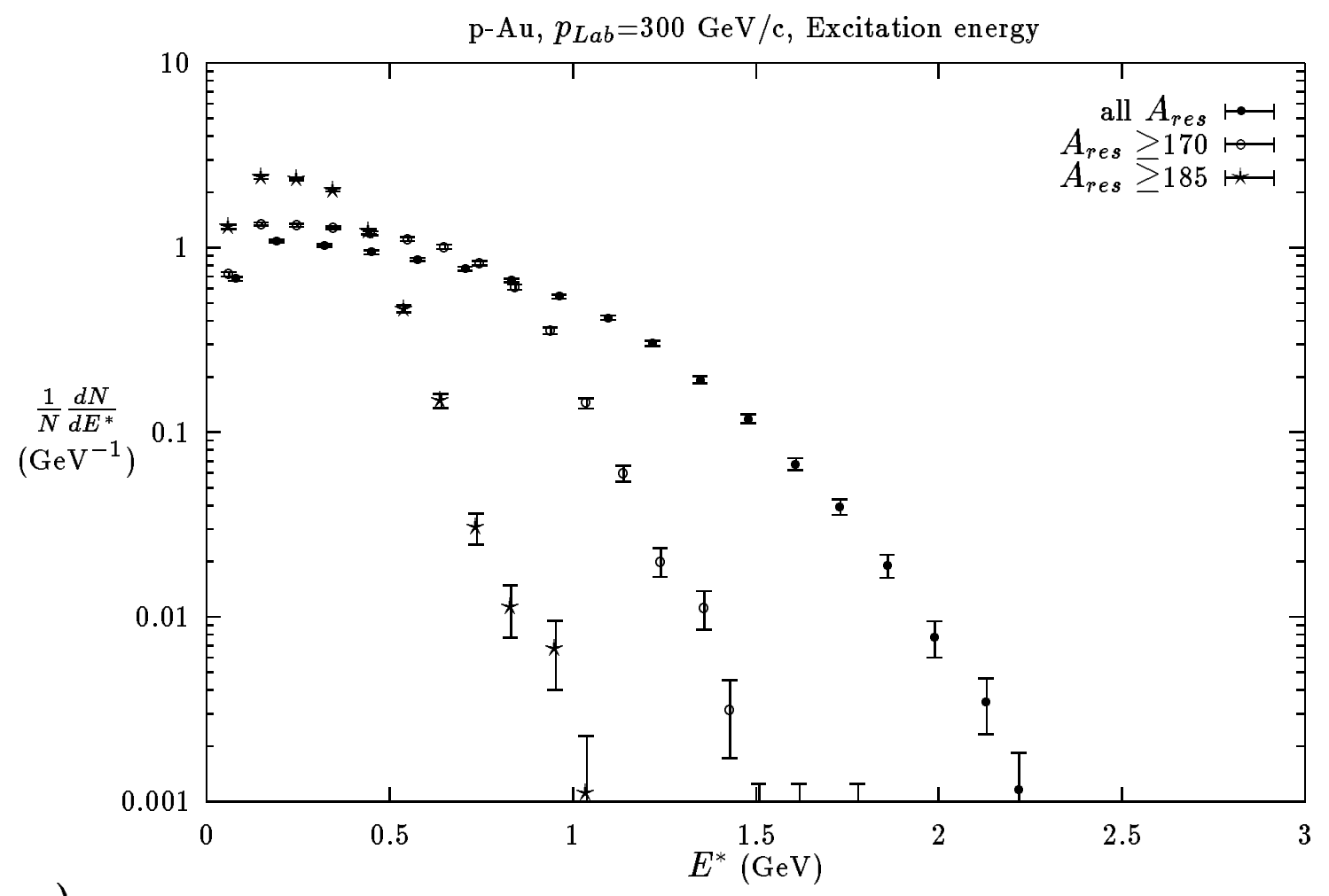

a)

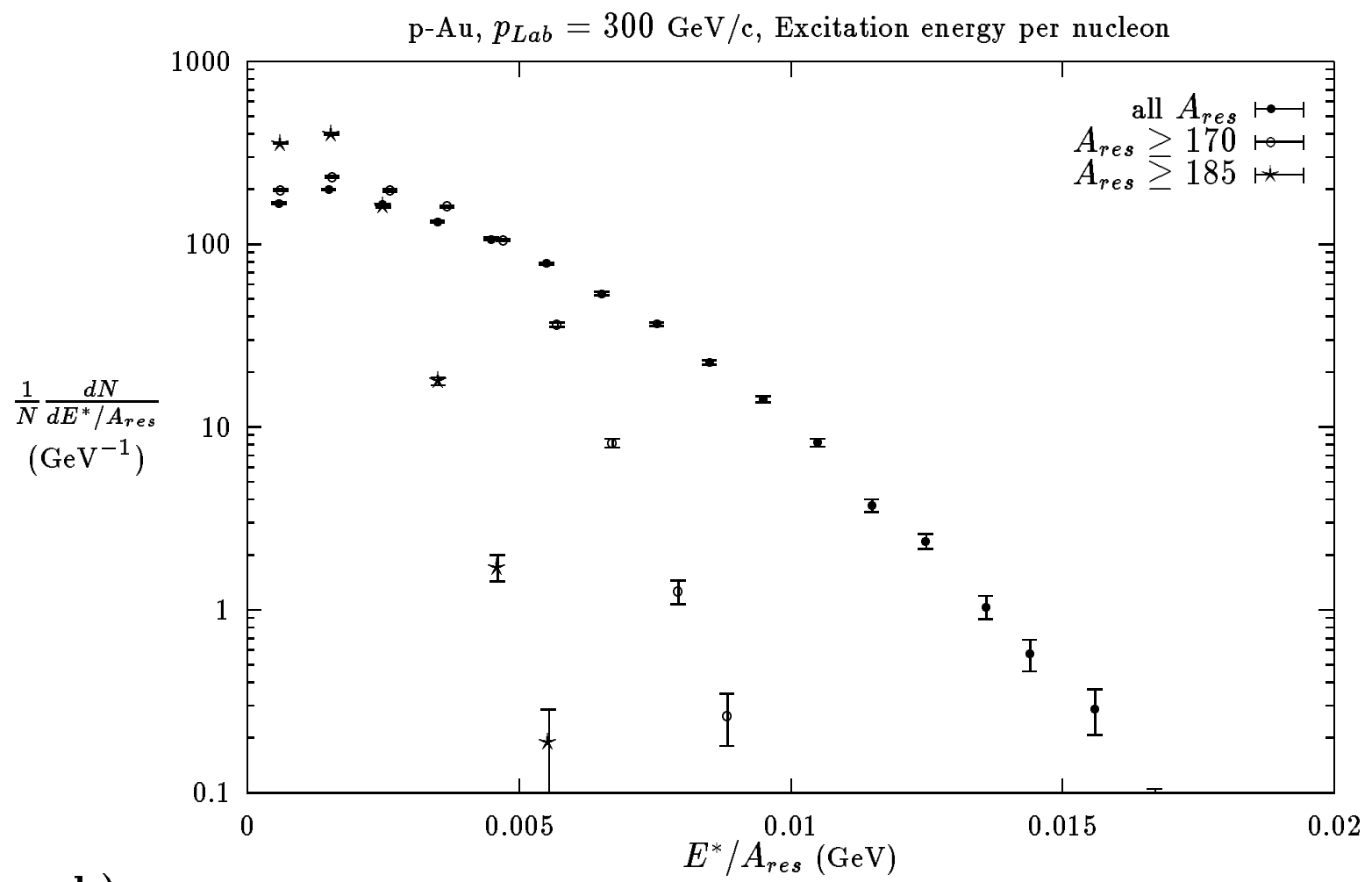

b)

Fig. 3 


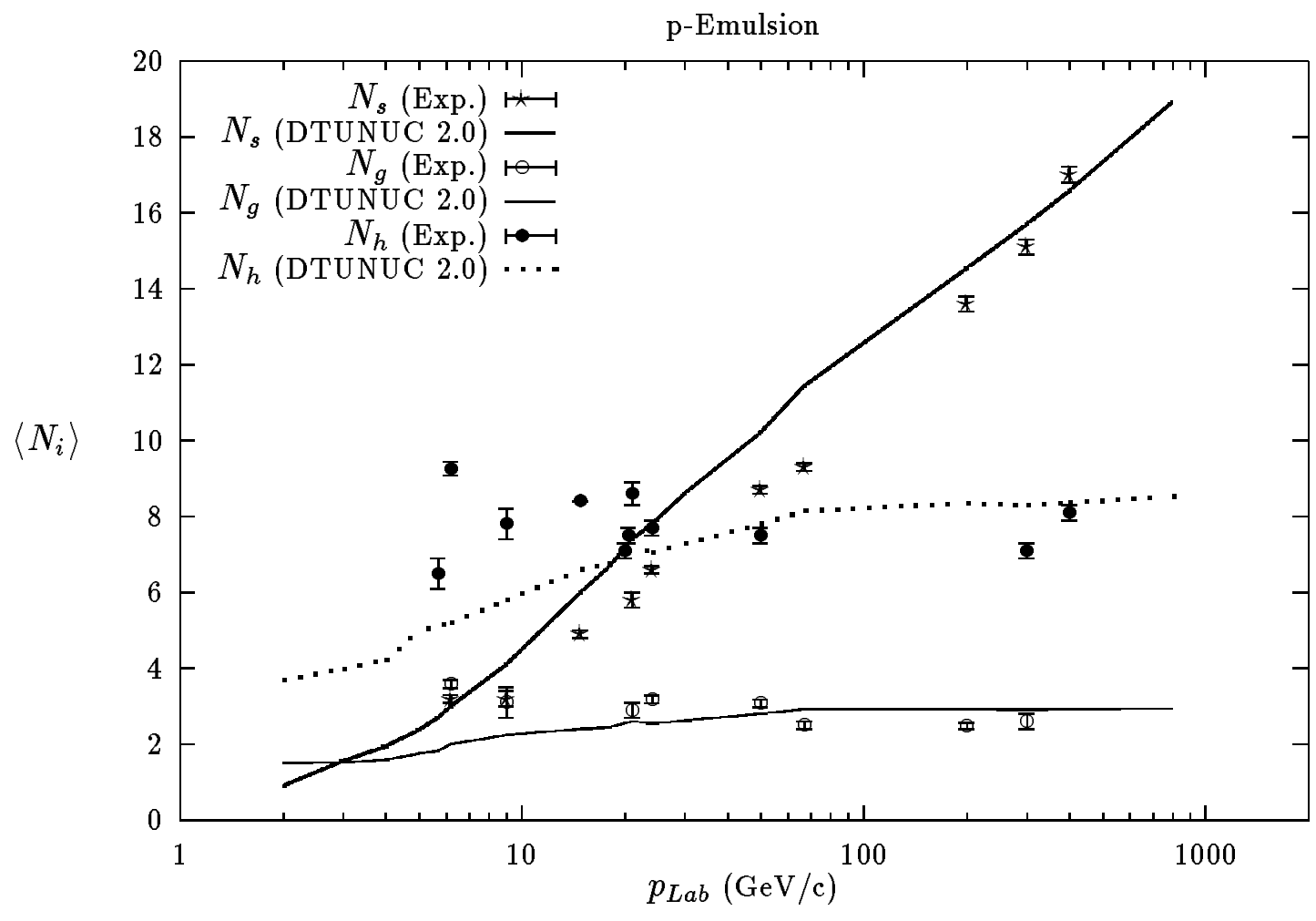

Fig. 4 


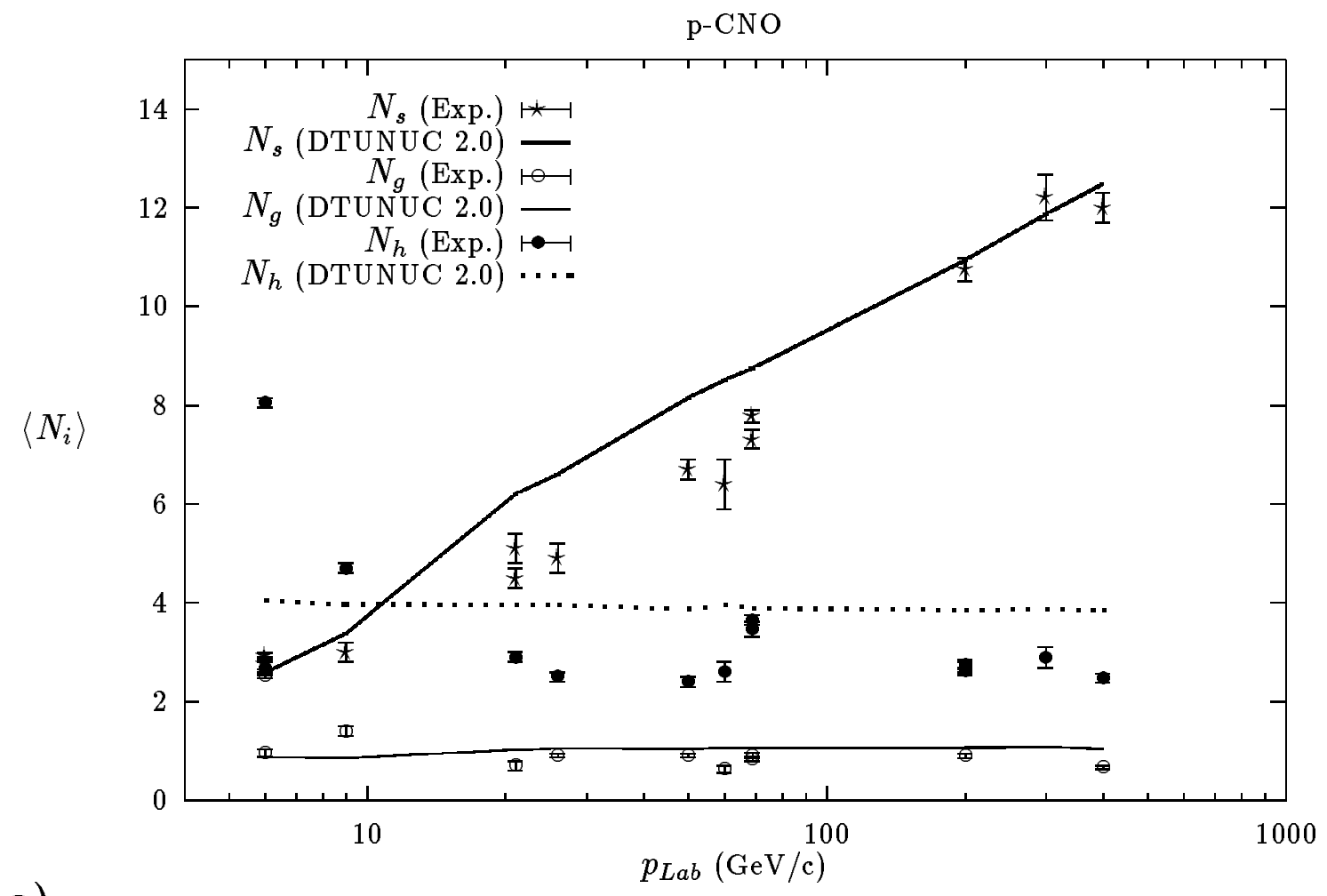

a)

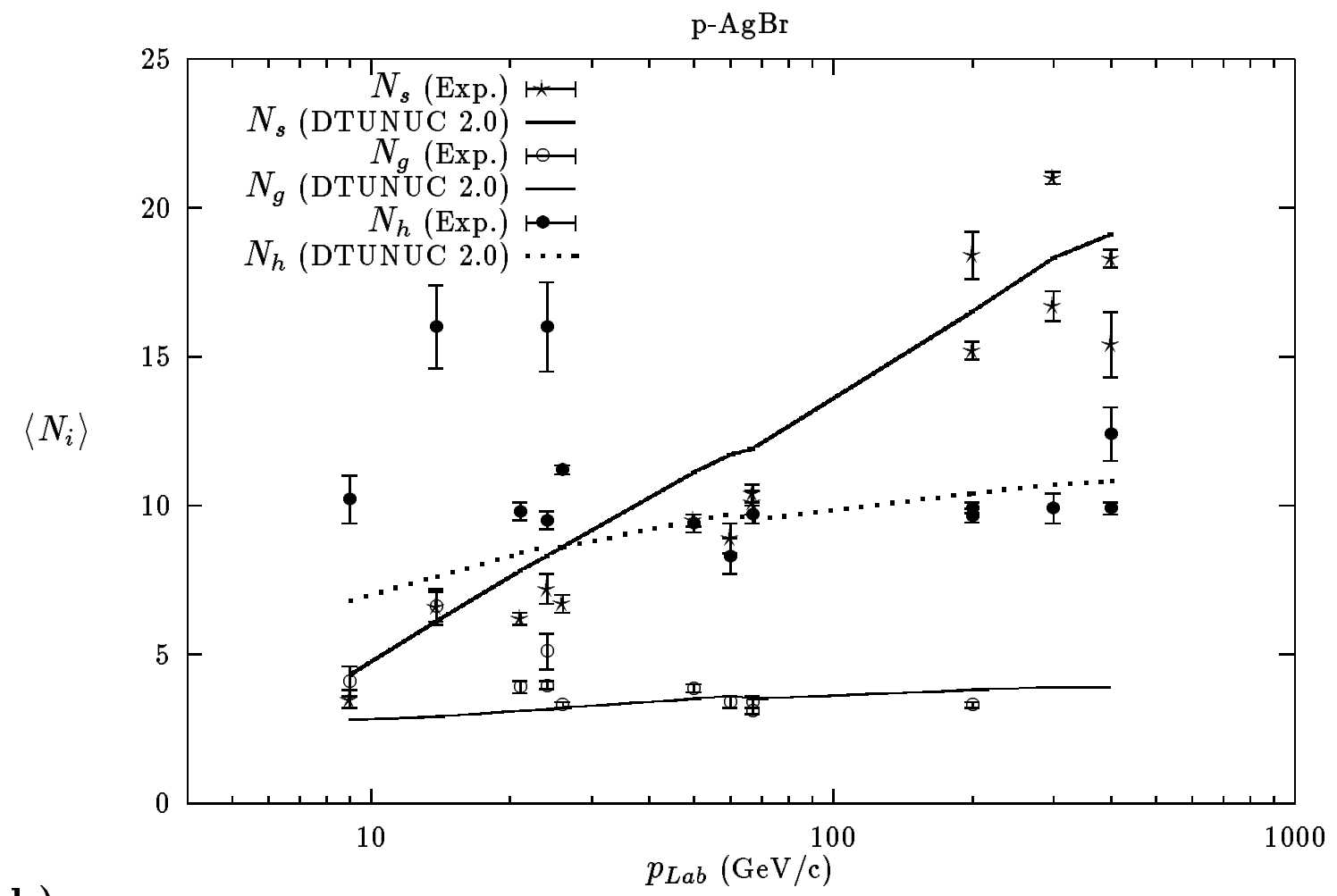

b)

Fig. 5 


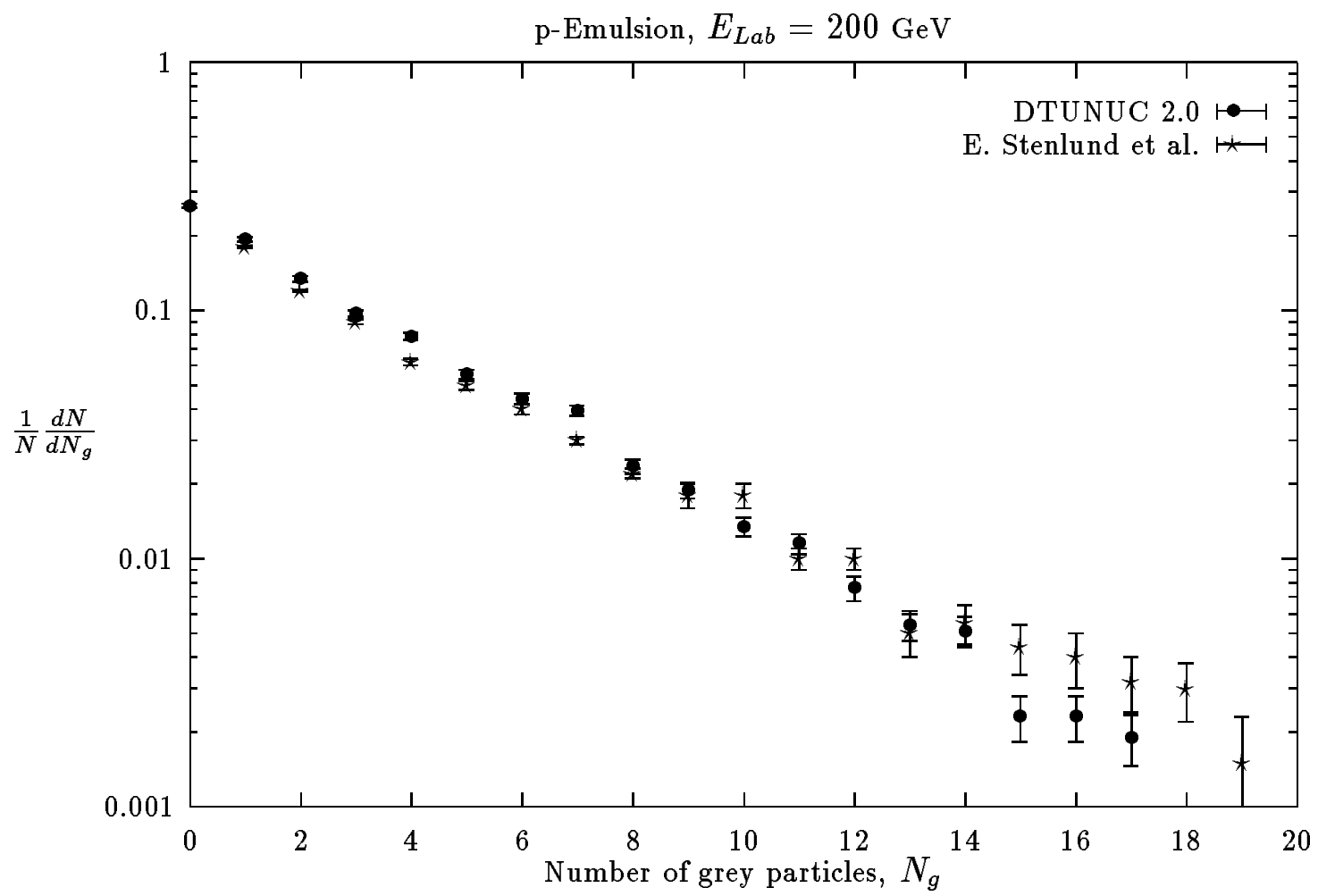

a)

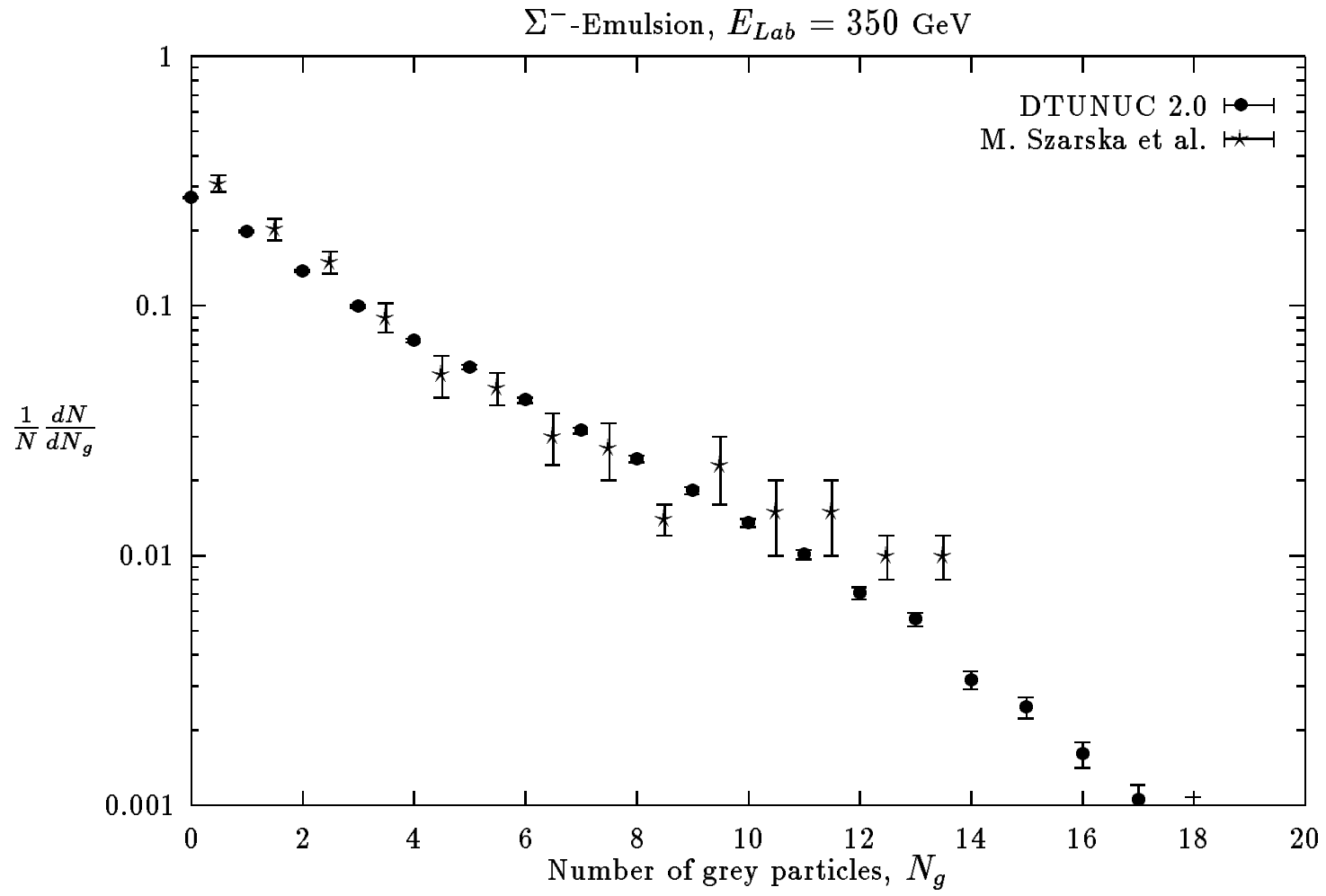

b)

Fig. 6 


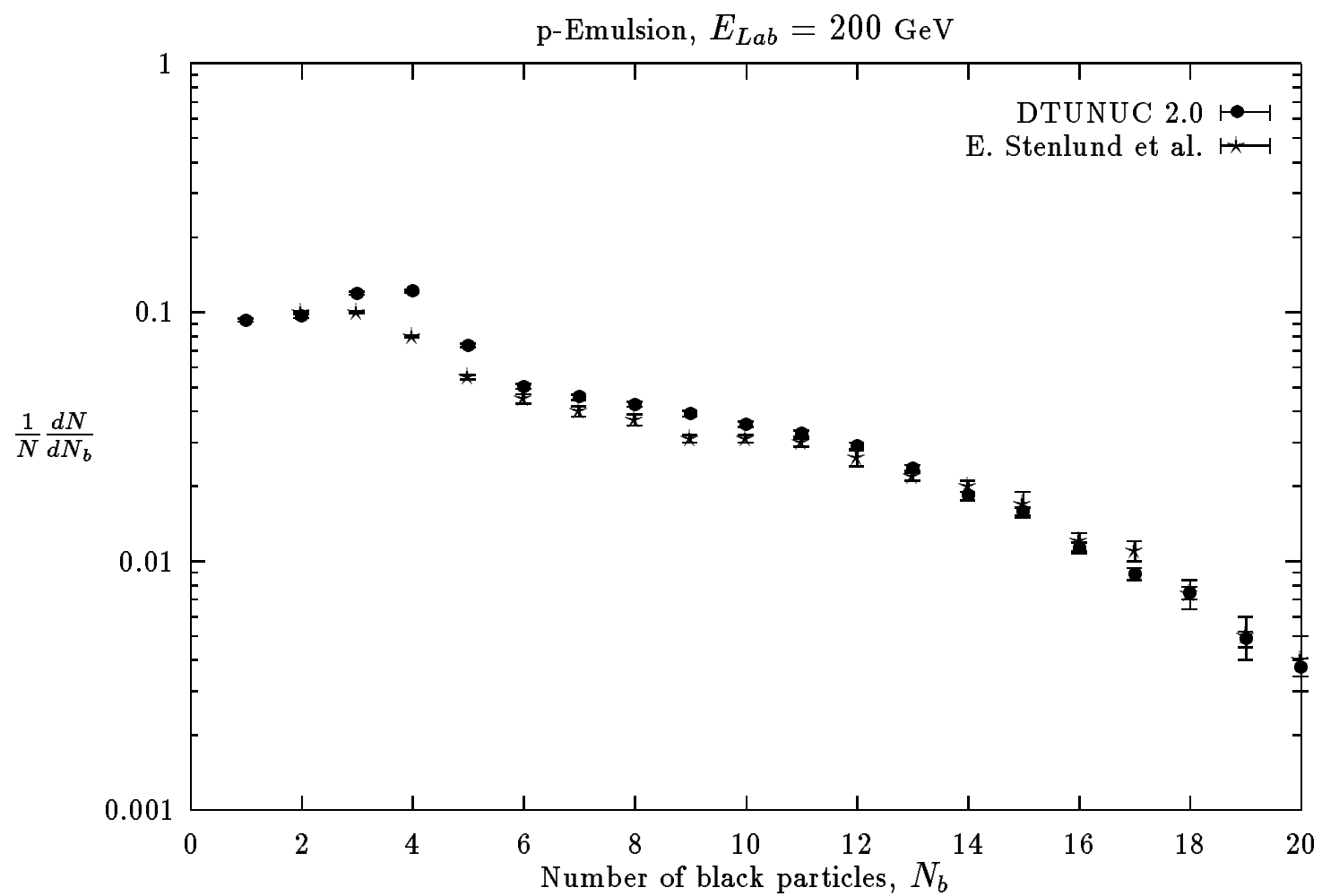

a)

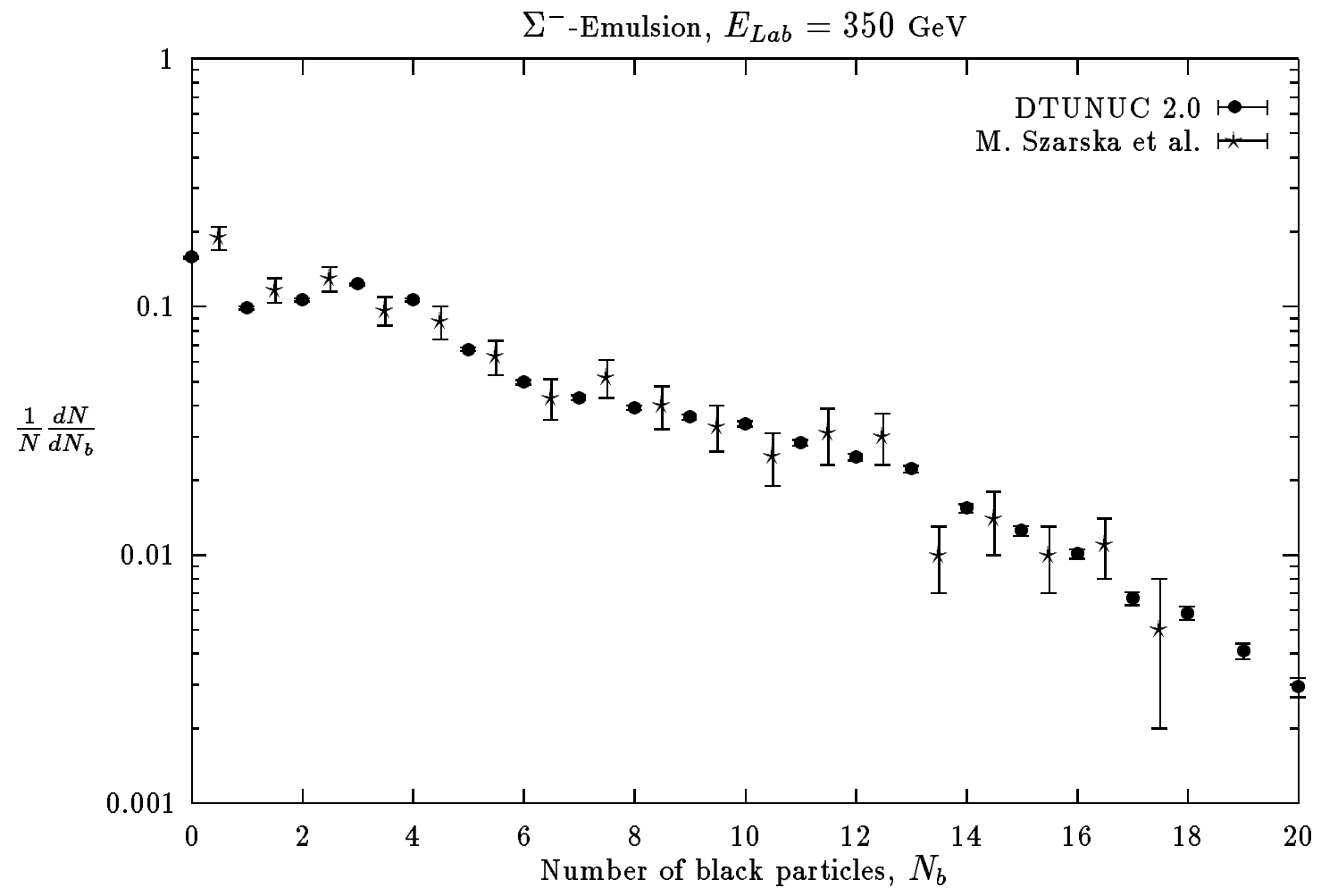

b)

Fig. 7 

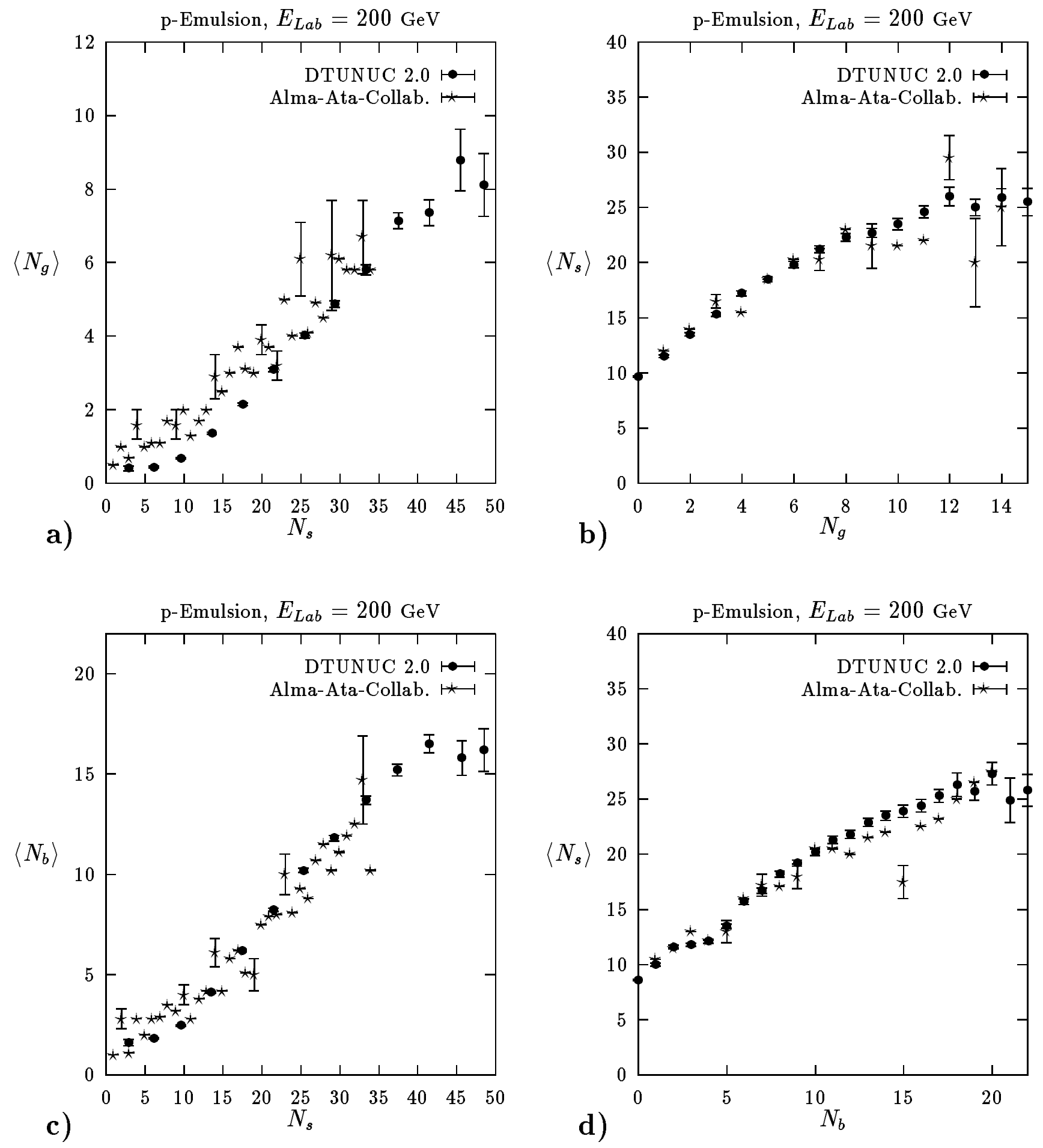

Fig. 8 

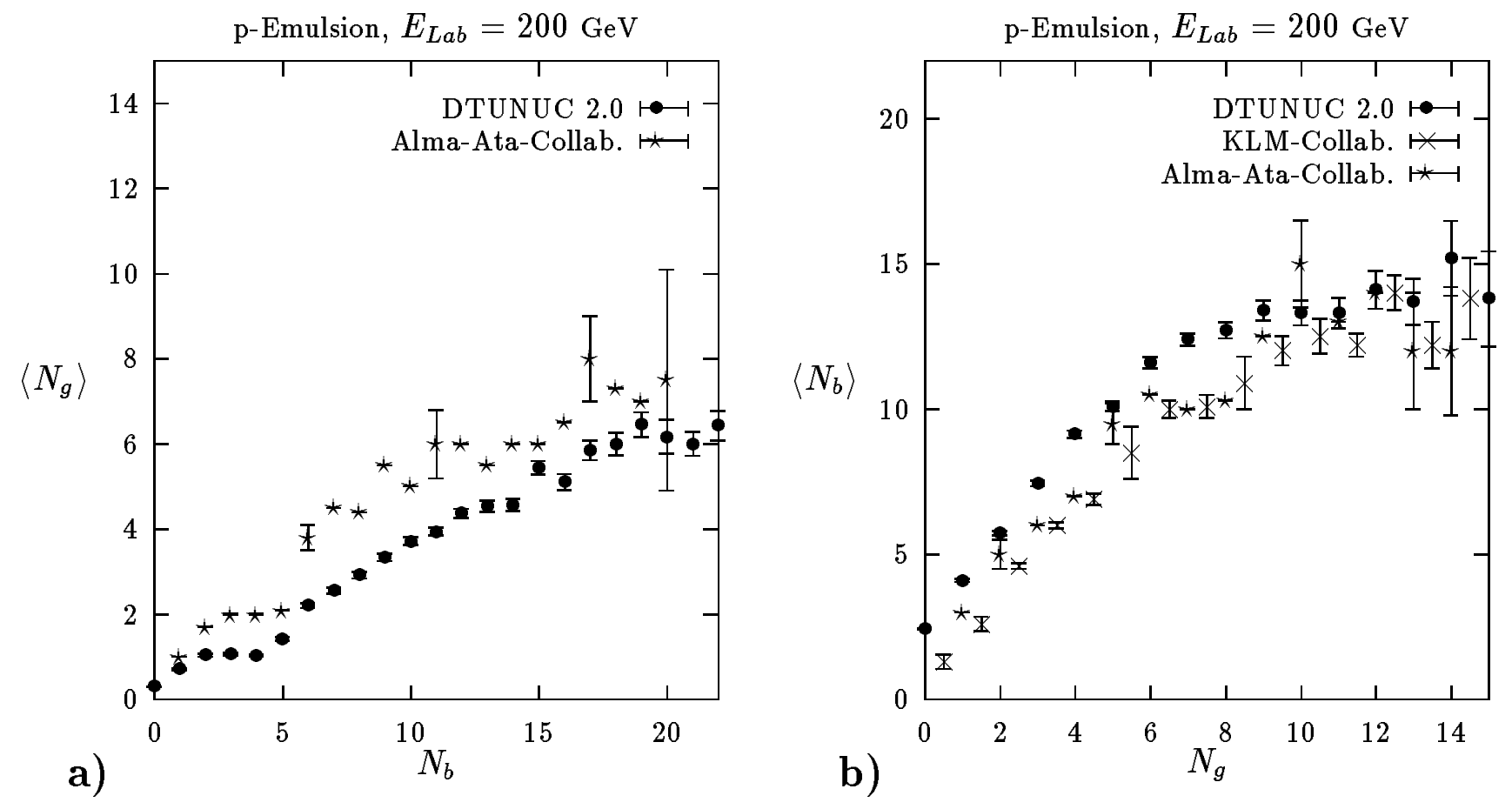

Fig. 9 


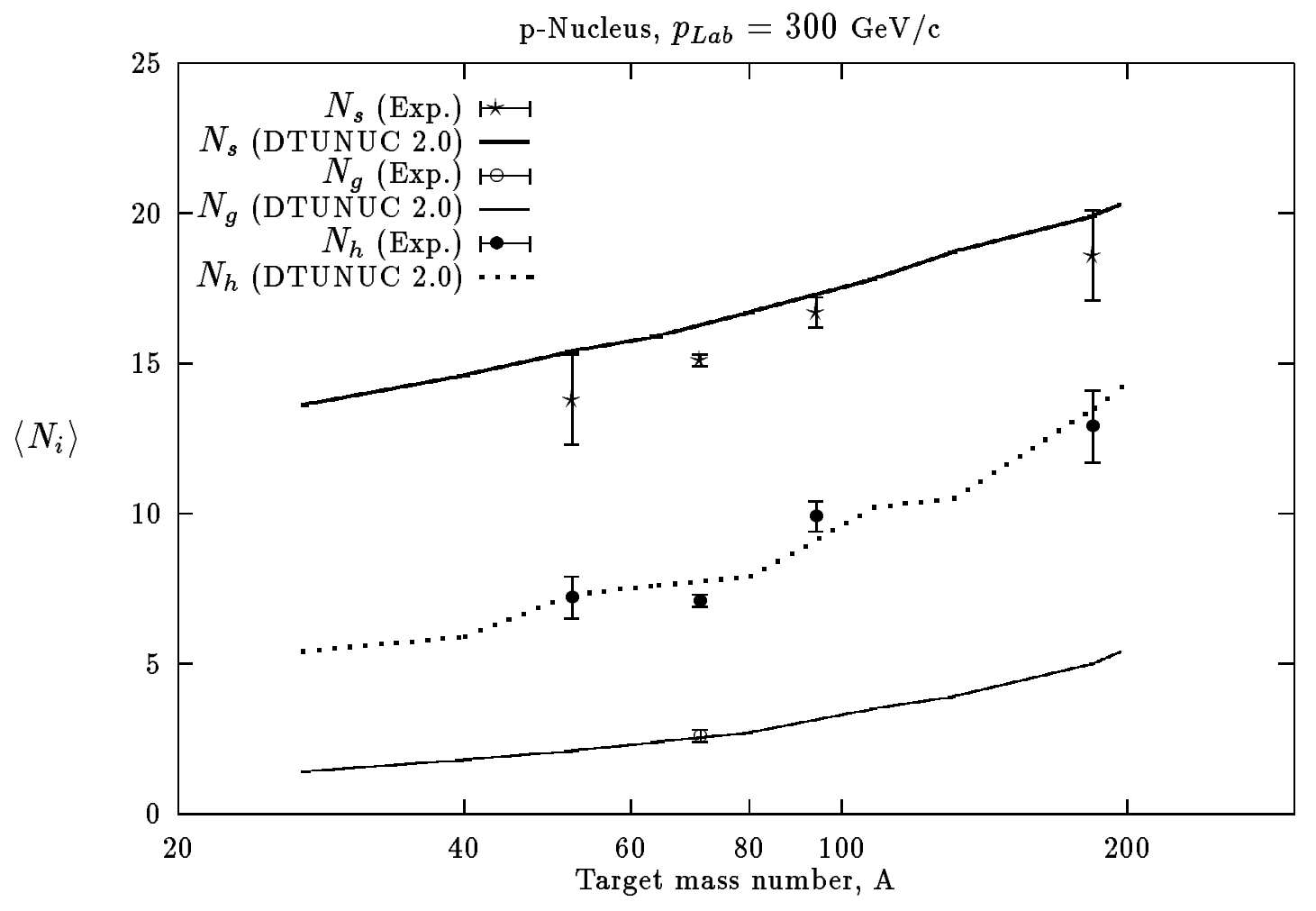

Fig. 10 


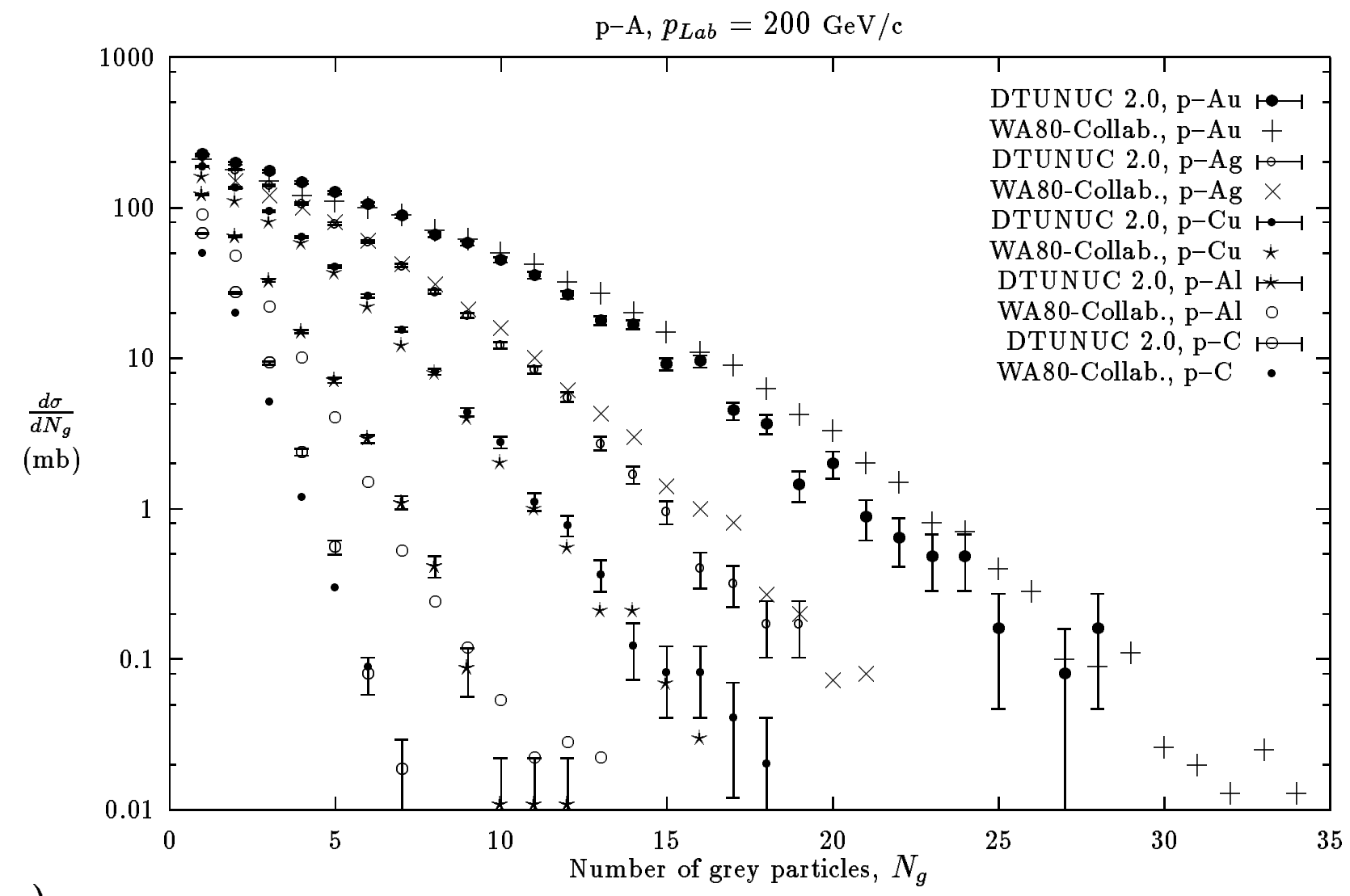

a)

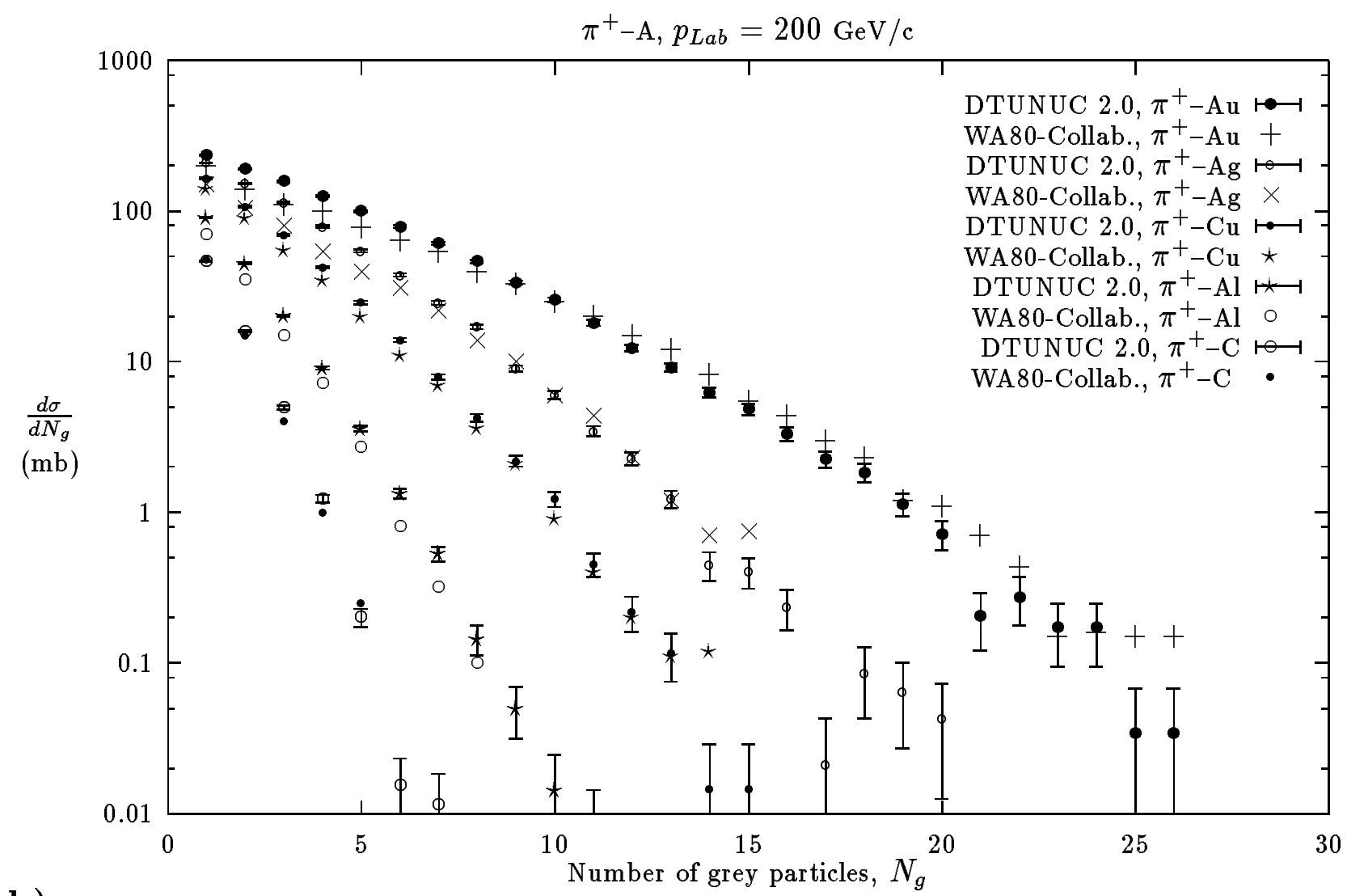

b)

Fig. 11 


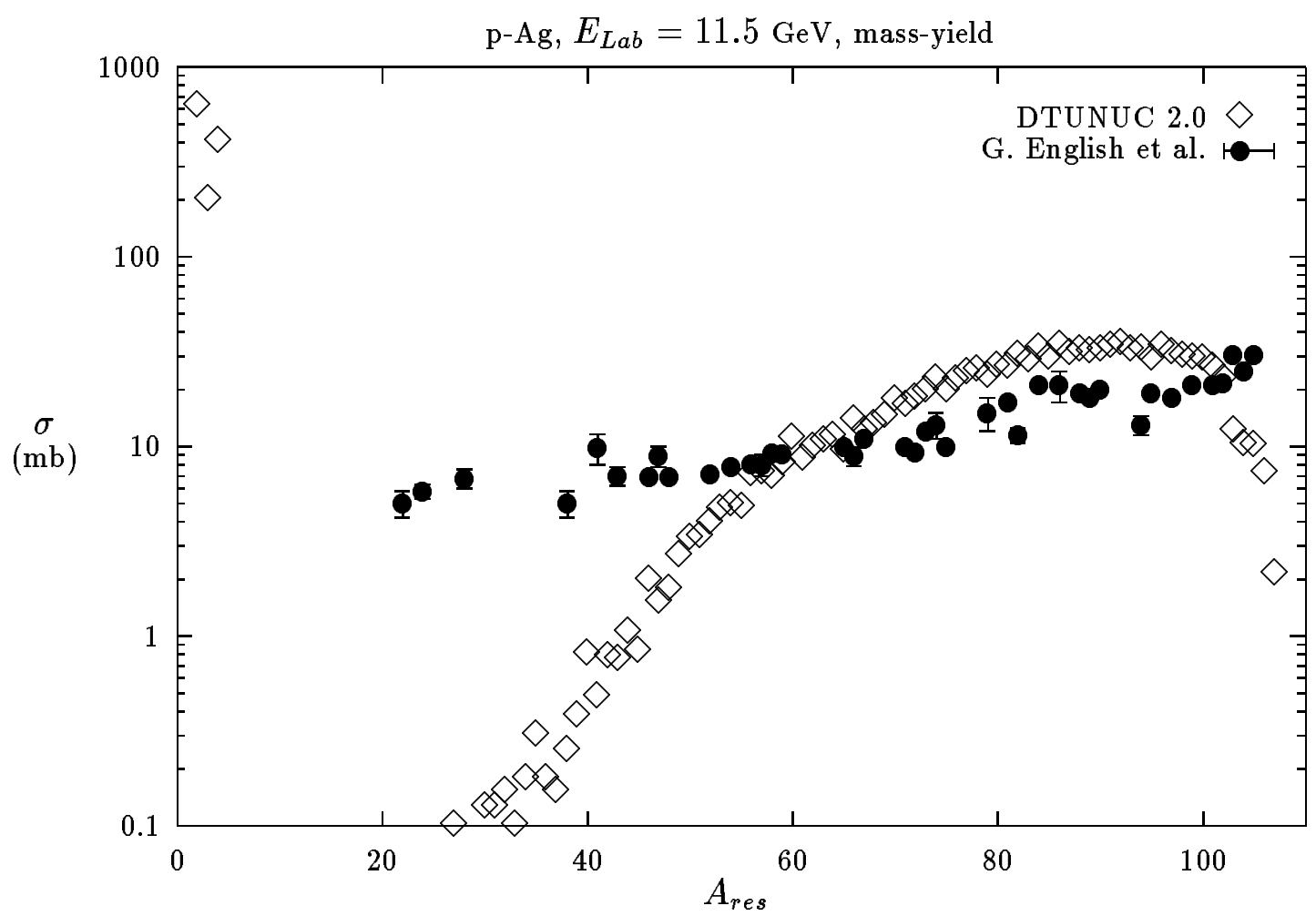

a)

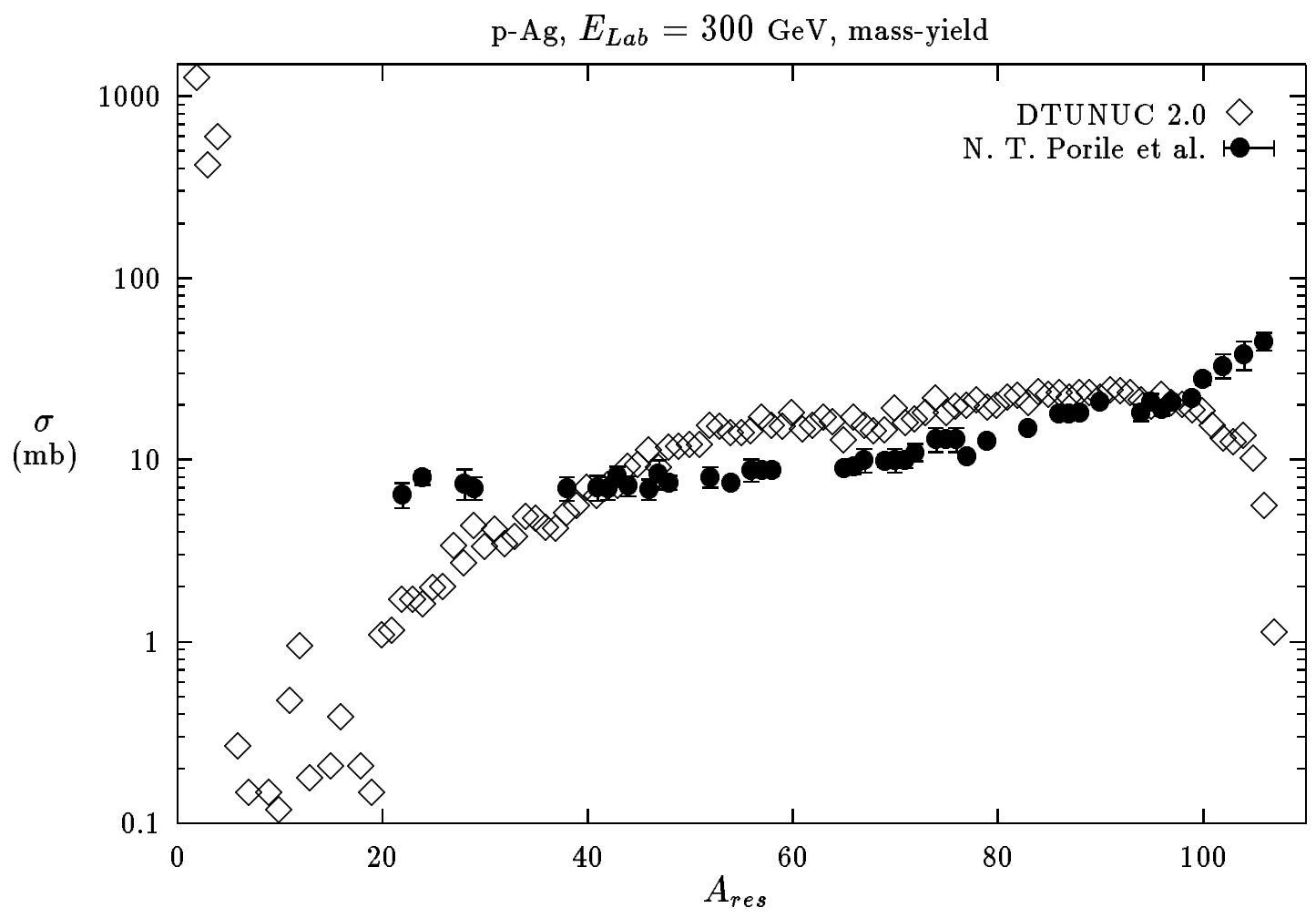

b)

Fig. 12 


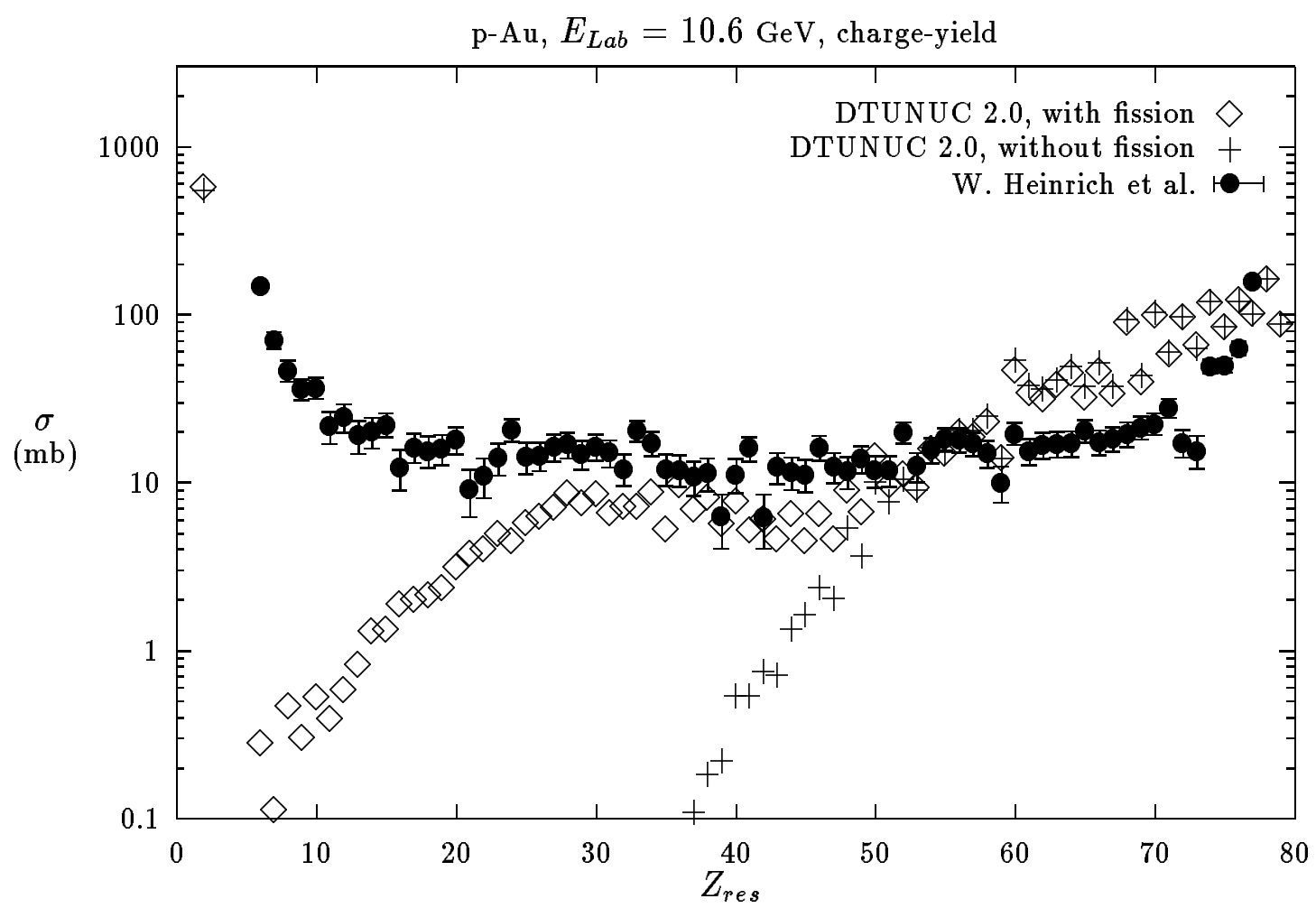

a)

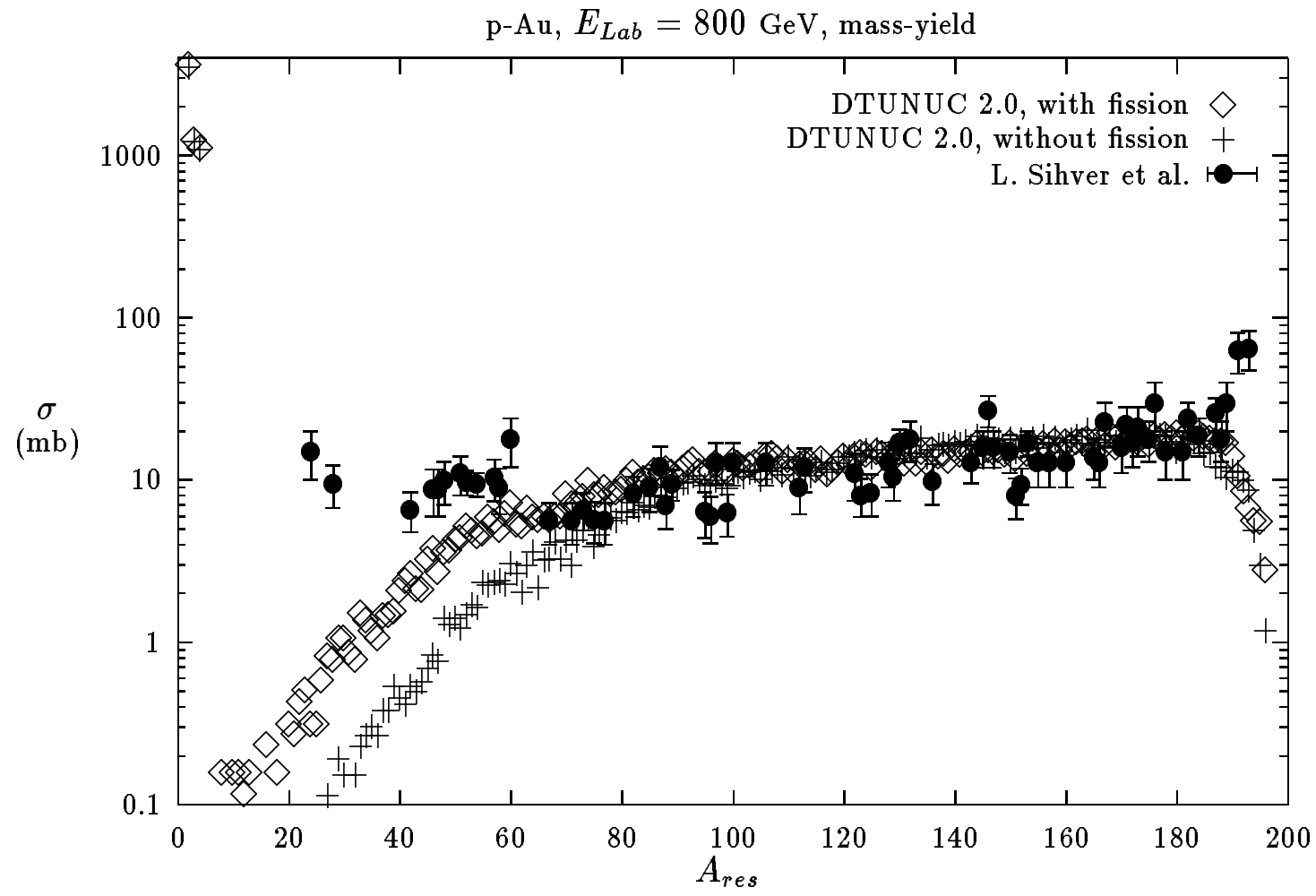

b)

Fig. 13 


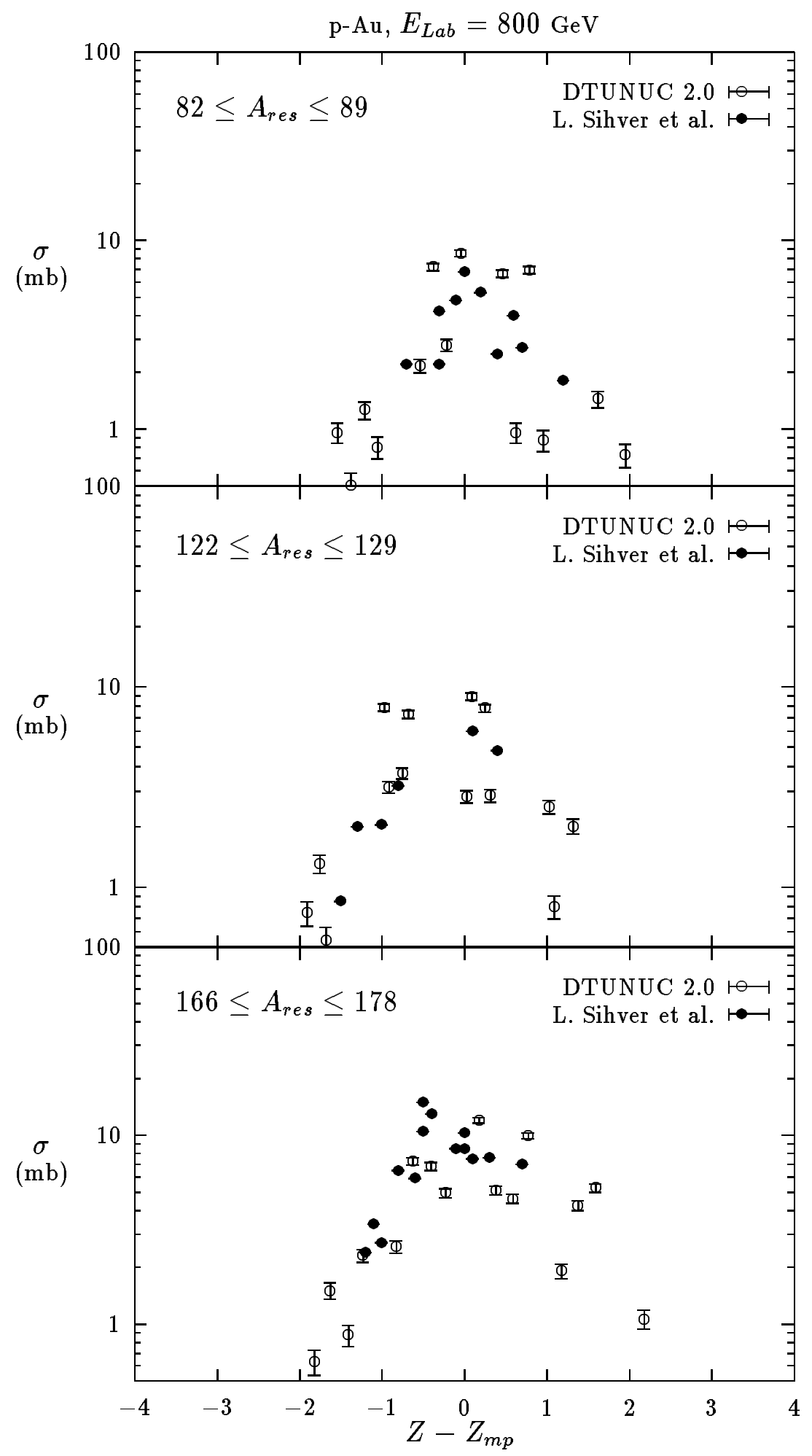

Fig. 14 


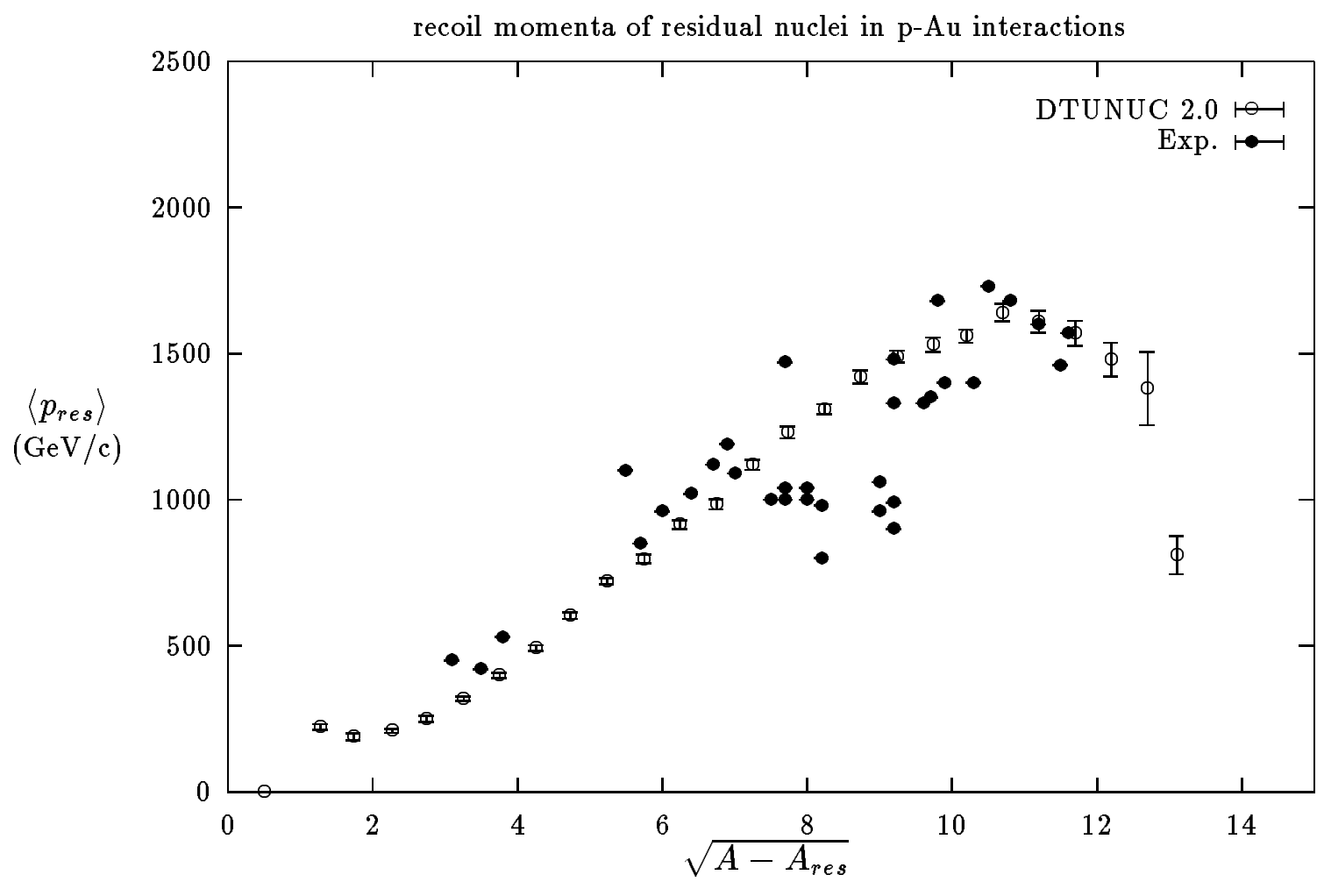

Fig. 15 